\title{
EXPECTATIVAS DE INFLACIÓN EN COSTA RICA
}

\author{
Alonso Alfaro Ureña ${ }^{1}$ \\ Carlos Monge Badilla ${ }^{2}$
}

Recibido: 20/01/2014

Aprobado: 05/01/2014

\begin{abstract}
RESUMEN
Como parte del proceso de transición hacia un esquema de Metas de Inflación (MI), el Banco Central de Costa Rica (BCCR) considera relevante caracterizar de la manera más completa posible las expectativas de inflación de la población, considerando tanto las particularidades de cada pronóstico que realizan como el proceso mediante el cual cada uno de los agentes incorpora la información disponible en la formulación de su expectativa individual sobre esta variable. El presente trabajo documenta el análisis de la información sobre expectativas de inflación contenida en la Encuesta Mensual de Expectativas de Inflación y de Variación del Tipo de Cambio. Se presentan diversas hipótesis sobre la formación de las expectativas, siendo las principales: expectativas racionales y expectativas adaptativas. Los resultados muestran sesgos importantes en las expectativas, así como una ausencia de aprendizaje respecto a los errores pasados por parte de los agentes consultados.

PALABRAS CLAVE: METAS DE INFLACIÓN, ENCUESTAS, BANCA CENTRAL, EXPECTATIVAS RACIONALES, EXPECTATIVAS ADAPTATIVAS.
\end{abstract}

\begin{abstract}
The Banco Central de Costa Rica (BCCR) is currently transitioning to an Inflation Targeting (IT) regime. In such context, it is relevant for the monetary authority to characterize in the most complete way possible the inflation expectations of the population, both in the particular elements of the forecasts and in the processes by which agents take information into account for their individual predictions. This paper documents the analysis of the inflation expectations data contained in the Monthly Survey of Inflation and Exchange Rate Expectations. We test several hypotheses such as rational and adaptive expectations. The results show significant biases in those expectations, while there is also a lack of learning from past errors by the agents surveyed.
\end{abstract}

KEY WORDS: INFLATION TARGETING, SURVEYS, CENTRAL BANKING, RATIONAL EXPECTATIONS, ADAPTATIVE EXPECTATIONS.

$1 \quad$ BCCR, Departamento de Investigación Económica, División Económica, Código Postal 10102, Costa Rica, alfaroua@bccr.fi.cr 


\section{INTRODUCCIÓN}

El Banco Central de Costa Rica (BCCR) ha manifestado públicamente su intención de implementar un sistema de Metas de Inflación (MI) con el objetivo de reducir en el mediano plazo la inflación interna a los mismos niveles de sus principales socios comerciales. La institución ha ejecutado políticas que contribuyen con dicho proceso, tales como anunciar regularmente en el Programa Macroeconómico un rango de meta explícito para el cambio interanual del nivel de precios, de la misma manera como se acostumbra en otros países en los que este esquema de política se ha implementado formalmente.

El éxito de un sistema de MI depende de que las expectativas que tienen los agentes respecto al cambio de los precios sean similares al cambio esperado y anunciado por el BCCR. Esto por cuanto expectativas con valores altos pueden generar una inflación mayor a la alcanzada, debido a que los agentes pueden tomar decisiones que generen inercia inflacionaria. Por lo tanto, resulta primordial en el cumplimiento de los objetivos de la institución conocer con detalle cómo se forman las expectativas de los agentes económicos, con el fin de comprender cómo la información externa y las propias acciones del BCCR afectan la toma de decisiones de los individuos ${ }^{3}$.

En Costa Rica se realizan dos encuestas que miden las expectativas de inflación de los agentes económicos. Hasta el momento, la información estadística que se recopila no ha sido explotada en todos sus alcances. Por ejemplo, Esquivel (2009) utiliza datos mensuales agregados para el análisis de la hipótesis de racionalidad y de cómo se forman las expectativas. Sin embargo, la base de datos disponible permite análisis más exhaustivos respecto a la heterogeneidad de los agentes, sus características y la evolución de sus percepciones a través del tiempo. La comprensión de dichos elementos, facilitada por la riqueza de la base de datos, representará un insumo valioso para la toma de decisiones de política monetaria.

El análisis de encuestas de expectativas de inflación ha sido un tema recurrente en la literatura. La profundidad del análisis es cada vez más relevante, ya que existen en la actualidad mejores herramientas para la medición de las expectativas de los agentes económicos. En el caso de los Estados Unidos de América (EUA), Mankiw, Reis y Wolfers (2004) exploran las características de diferentes encuestas de expectativas de inflación disponibles, encontrando que el nivel de desacuerdo en las expectativas entre los agentes es significativo y que esa heterogeneidad también depende de los valores que tomen diferentes agregados macroeconómicos.

Un caso más cercano a la experiencia costarricense es el de Uruguay. Borraz y Gianelli (2011) realizan un análisis de la encuesta de expectativas y encuentran que el poder predictivo de los analistas a quienes se les realiza la consulta es bajo. Además, los pronósticos individuales tienden a converger en el tiempo hacia la media que se divulga cada mes. Aunado a esto, es notorio que el límite superior del rango de la meta tiene una importancia relevante para la realización de los pronósticos, mientras que los instrumentos de política monetaria no la tienen.

En ambos estudios se realizan pruebas de hipótesis para verificar el uso que se le da a la información económica disponible en el momento de generar la expectativa. Para esto, se analiza mediante regresiones si los errores de predicción están correlacionados con el comportamiento de variables relevantes al momento de realizar el pronóstico, como el nivel de desempleo, la inflación y los bonos del tesoro, entre otros. Además de someter a prueba la hipótesis de que sí existen sesgos en los pronósticos, se demuestra que los errores de predicción son persistentes en el tiempo.

Leyva Jiménez (2012) realiza, para Guatemala, un análisis exhaustivo de las hipótesis de racionalidad y optimalidad de los pronósticos para un panel pequeño de analistas privados a los cuales se les consulta sobre la expectativa de inflación para varios horizontes. Este estudio encuentra que estos pronósticos no son óptimos, y son susceptibles de mejoras sustanciales.

3 En línea con esto, el Departamento de Investigación Económica (2012) ha planteado como un tema en su plan bianual de investigación el análisis de las encuestas de expectativas que realiza el BCCR. 
Finalmente, el autor sugiere algunos elementos para mejorar paulatinamente la información con que cuentan los analistas sobre los pronósticos que ellos mismos realizan, una práctica que es común en otros países.

Es relevante para el presente estudio analizar por separado el periodo que identifica Torres (2012), a partir del cual existe evidencia de un cambio estructural para la inflación de Costa Rica. Precisamente, es a partir de mayo de 2009 que las variaciones interanuales del IPC se redujeron a un dígito, $y$ mantuvieron durante los años siguientes $y$ hasta la actualidad niveles que rondan el 5\%. Sin duda, este es el periodo de mayor interés para el presente estudio, al ejemplificar la lenta convergencia de las expectativas de inflación al nivel efectivo de inflación, aun cuando esta última no ha tenido variaciones importantes ${ }^{4}$.

Este documento se organiza en cuatro partes. En la segunda parte se profundiza sobre los determinantes de la formación de las expectativas de los agentes económicos, mediante distintos métodos analíticos. La tercera parte propone un modelo base para proyectar los datos de expectativas de inflación en el corto y mediano plazo, mientras que la última parte presenta las conclusiones $y$ recomendaciones.

A continuación se explica la naturaleza de las bases de datos utilizados en este estudio.

\section{Datos utilizados}

Tal y como se mencionó previamente, el BCCR realiza dos encuestas cuyo objetivo es capturar las expectativas de inflación de los agentes económicos: la Encuesta Mensual de Expectativas de Inflación y de Variación del Tipo de Cambio y la Encuesta Trimestral Perspectivas Económicas, elaboradas por el Departamento de Gestión de la Información Económica (DGIE) de la División Económica (DE) del BCCR. La encuesta trimestral arroja resultados muy similares a la encuesta mensual, por lo que este documento se concentrará en analizar la información de la encuesta mensual, con el fin de aprovechar las ventajas de una mayor cantidad de observaciones ${ }^{5}$.

Es relevante destacar que un dato obtenido a través de la encuesta en un mes y año particular, debe ser interpretado como la expectativa de la inflación interanual que se presentará once meses después. Esto por cuanto los agentes formulan su expectativa para doce meses, pero son consultados al respecto en el transcurso del primer mes de ese periodo anual que están pronosticando ${ }^{6}$. Por ejemplo, aquellas expectativas manifestadas en enero tratan de pronosticar la inflación a diciembre de ese mismo año. Por lo tanto, el dato recopilado durante enero debe ser comparado con la inflación observada 11 meses después (diciembre).

Los datos de la encuesta mensual inician en enero de 2006, cuando diciembre de 2006 era la primera fecha para la cual existe una medición del sesgo de las expectativas. Se cuenta con disponibilidad de datos hasta abril de de 2013, por lo que hay 77 meses en la muestra para todos los cál-

$4 \quad$ Alternativamente se consideró utilizar como punto de división de los periodos el mes en el que el promedio de las expectativas se reduce a un dígito. A pesar de existir algunas diferencias cuantitativas, los resultados cualitativos son sumamente similares. Las únicas excepciones se discutirán cuando se evalúen diferentes hipótesis de racionalidad. Se prefirió utilizar la división en mayo 2009 por existir investigación previa que respalde esta elección, además de ser el quiebre estructural propio de la variable sobre la cual se forman las expectativas.

$5 \quad$ En el gráfico 1 se puede observar como ambas encuestas presentan resultados muy similares en cuanto a su nivel y evolución. El comportamiento de la serie de expectativas recopilada trimestralmente, desagregada por estratos, también es muy similar a su correspondiente mensual.

6 A pesar de que la proyección se realiza durante el primer mes del periodo pronosticado, la misma se debe interpretar para un año calendario, ya que los agentes desconocen el dato de la inflación que ocurre para este primer mes. Esto debido a que el dato de inflación es publicado por el Instituto Nacional de Estadística y Censo (INEC) hasta los primeros días del siguiente mes. 
culos que requieran datos de cuán acertado fue un pronóstico. Para datos descriptivos, la muestra aumenta en 11 meses.

En la base de datos utilizada para el presente estudio hay 108 individuos, a los que a lo largo del periodo muestral se les ha consultado en algún momento sobre su expectativa de inflación. En los primeros meses para los cuáles se recogieron datos, la muestra consistía de alrededor de 27 personas mientras que ese número se ha duplicado y ronda los 60 individuos consultados en los primeros meses del año 2012 (la expectativa relevante para los meses recientes).

La muestra está dividida en cuatro estratos: analistas bursátiles (60\%), consultores (21\%), académicos (11\%) y empresarios (8\%). Es posible, por lo tanto, conocer el poder predictivo de cada uno de los grupos consultados así como darle seguimiento a cada uno de los individuos que se haya mantenido en la muestra a lo largo de un periodo extendido.

En junio de 2012 se dio un cambio en el tamaño y la composición de la muestra. El número de individuos consultados se duplicó, pasando a 119, además que el estrato empresarial pasó a ser el más numeroso. Durante los dos meses en los cuáles se realizaron las consultas, para ambas muestras, los resultados son sumamente similares ${ }^{7}$. No obstante, es de suma relevancia comprender las particularidades del comportamiento histórico del estrato de empresarios, ya que será la dinámica de este grupo la que determinará en mayor medida el comportamiento a futuro de la serie agregada que se publica.

\section{ANÁLISIS DE LAS ENCUESTAS DE EXPECTATIVAS DE INFLACIÓN}

\section{Caracterización y metodología}

Costa Rica ha experimentado cambios recientes sustanciales en las magnitudes de la variación interanual del Índice de Precios al Consumidor (IPC). Después de poco más de treinta años, en los cuales la inflación medida a diciembre de cada año usualmente tenía valores cercanos o superiores al 10\%, los años 2009-2011 han finalizado con cifras en el rango de 4\%-6\%.

El proceso mediante el cual el promedio de las expectativas finalmente se ha alineado con el rango de meta de inflación establecido por el BCCR no ha sido continuo ${ }^{8}$. Después de la flexibilización del mercado cambiario y los primeros anuncios de rangos de meta para la inflación, las expectativas rápidamente se ubicaron en el rango de 8\%-10\%, pero hubo cambios significativos en las expectativas de los agentes económicos que se generaron posterior al periodo inflacionario de 2008-2009. A partir de ese momento ha existido una desalineación de esas mismas expectativas con respecto a los datos efectivos de la inflación interanual que solamente se revirtió en los últimos meses por la aceleración reciente de la inflación, tal y como se puede apreciar en el Gráfico 1.

$7 \quad$ Adicionalmente, las expectativas recopiladas con la nueva muestra solo podrán ser evaluadas a partir de junio de 2013, por lo que el impacto de los nuevos resultados en los análisis de sesgo en las expectativas aún no son medibles.

8 A partir de julio del 2012 y hasta febrero de 2013 la expectativa promedio se mantuvo dentro del rango meta de 4\%-6\%, mientras que para marzo y abril de 2013 esta variable alcanzó un nivel de 6,4\%. 

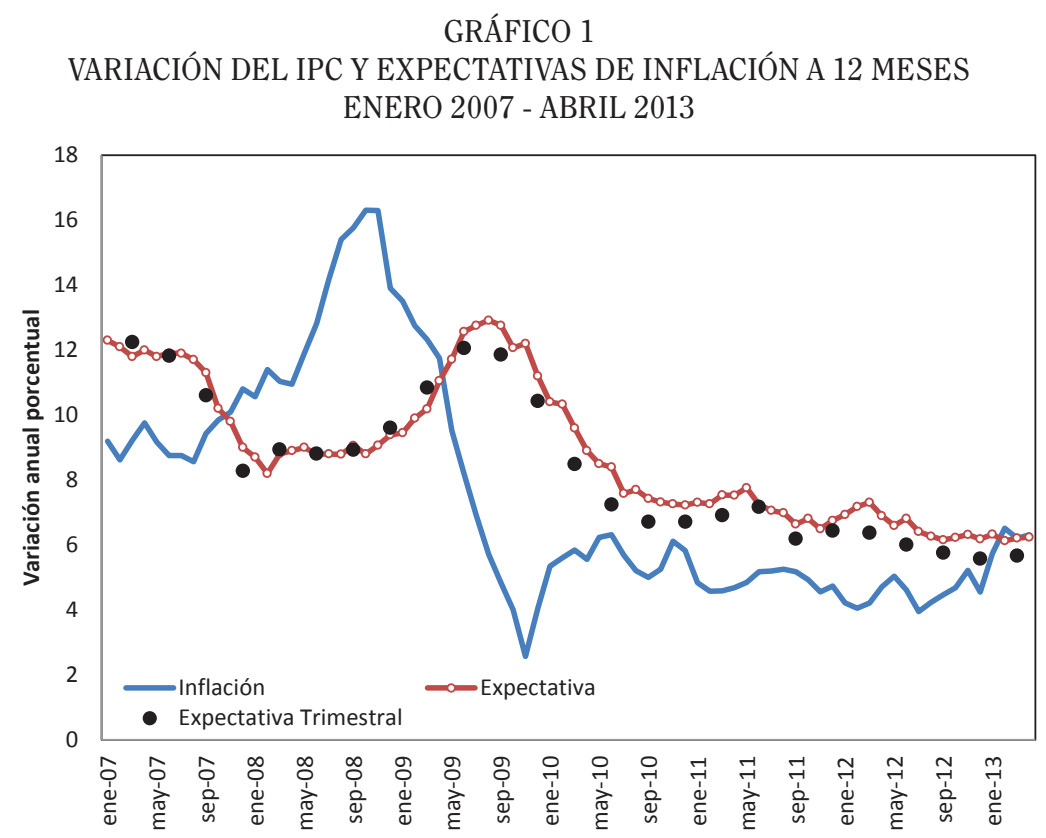

Fuente: Elaboración propia.

Una de las contribuciones del presente artículo es el uso de la base de datos de la encuesta de expectativas de inflación desagregada por individuo. Usualmente las hipótesis que se someten a prueba al utilizar este tipo de encuestas suponen implícitamente la existencia de un agente representativo de la población. Esto significa que el promedio de los criterios individuales se toma como el criterio poblacional y, a partir del mismo, se realizan los análisis deseados de hipótesis de racionalidad, sesgos en los pronósticos y otros. Keane y Runkle (1990) resaltan que esto es incorrecto, ya que genera un sesgo de agregación importante. Por ejemplo, basta tomar una ecuación sencilla de la siguiente manera:

$$
\pi_{t}=a+b * E_{i, t-12} \pi_{t}
$$

donde $E_{i, t-12} \pi_{t}$ representa la expectativa que el individuo $i$ manifiesta en $t-12$ para la inflacion $\pi$ del periodo $t$.

La hipótesis de que no existen sesgos en los pronósticos se puede resumir en probar que $a=0$ y $b=1$ simultáneamente. Sin embargo, existen al menos tres formas diferentes mediante las cuales los coeficientes pueden ser estimados para una población. En primer término, se puede realizar la estimación de la Ecuación (1) para cada individuo, y posteriormente se puede obtener un promedio de todos los coeficientes estimados. Por otro lado, se puede tomar todos los datos agrupados y estimar una sola regresión con muchas observaciones (todos los agentes y todos los meses incluidos en la muestra). También se puede, tal y como es usual, obtener el promedio mensual de las expectativas de los individuos de la población y, con ello, estimar una sola ecuación con una observación para cada mes observado.

Los autores muestran que los coeficientes estimados en cada caso, respectivamente, tendrán la siguiente forma: 


$$
\begin{aligned}
& \hat{b}_{i}=\frac{1}{N} \sum_{i=1}^{N} \frac{\sum_{t=1}^{T} \pi_{t} * E_{i, t-12} \pi_{t}}{\sum_{t=1}^{T}\left(E_{i, t-12} \pi_{t}\right)^{2}} \\
& \hat{b}_{a}=\frac{\sum_{i=1}^{N} \sum_{t=1}^{T} \pi_{t^{*}} E_{i, t-12} \pi_{t}}{\sum_{i=1}^{N} \sum_{t=1}^{T}\left(E_{i, t-12} \pi_{t}\right)^{2}} \\
& \hat{b}_{p}=\frac{\sum_{t=1}^{T} \pi_{t}\left(\sum_{i=1}^{N} E_{i, t-12} \pi_{t}\right)}{\sum_{t=1}^{T}\left(\sum_{i=1}^{N}\left(E_{i, t-12} \pi_{t}\right)^{2}\right)}
\end{aligned}
$$

donde $i \in 1, \ldots, N$ y $t \in 1, \ldots, T$. El promedio de los coeficientes individuales (i) y el coeficiente de los datos agregados $(a)$ convergen en probabilidad al mismo valor, pero $\frac{\hat{b}_{a}}{\hat{b}_{p}}$ toma, necesariamente, un valor menor a 1. Esto significa que, siempre que los pronósticos de los individuos difieran, el utilizar los datos promediados (p) de las expectativas como las observaciones para la estimación, le impondrá un sesgo positivo a los coeficientes estimados.

Un ejemplo donde se utilicen los datos para el caso costarricense puede ayudar a cuantificar la magnitud del sesgo que podría tener en el análisis que se presentará posteriormente. Si se utilizan datos a partir de mayo 2009 (fecha para la cual está identificado el quiebre estructural e inician los sesgos hacia la sobreestimación de la inflación) y hasta junio de 2012 (último mes en el que las expectativas se mantuvieron ininterrumpidamente por encima del límite superior del rango meta) y se realizan las regresiones para los 71 individuos para los cuales existe información, $\hat{b}_{i}=0,11, \hat{b}_{a}=0,10$ y $\hat{b}_{p}=0,16$. El sesgo de alrededor de un $45 \%$ de la magnitud del parámetro es similar al reportado por Urich yWachtel (1984) para el caso estadounidense.

El gráfico 2 muestra un histograma de las expectativas para dos meses, uno al inicio y otro al final del periodo muestral ${ }^{9}$. En él es posible apreciar que la distribución de las expectativas es

GRÁFICO 2

HISTOGRAMA DE LAS EXPECTATIVAS
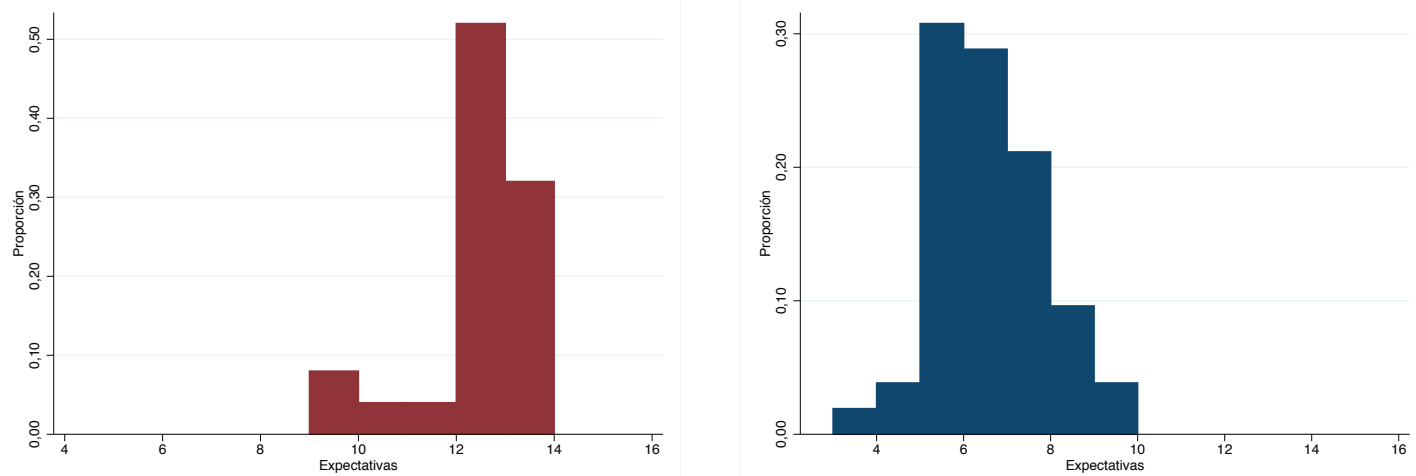

Fuente: Elaboración propia.

9 La expectativa para enero de 2007 fue recolectada en febrero de 2006, mientras que la de abril de 2013 fue recolectada en mayo de 2012. 
asimétrica en direcciones opuestas. Adicionalmente (aunque no se refleja en estos dos gráficos), hay varios meses para los cuales la distribución es bimodal, lo cual incrementa los problemas de suponer que el promedio sea representativo de la población para cuantificar los sesgos individuales.

Por lo tanto, esto sugiere que los análisis que se realicen de las encuestas de expectativas de inflación deben utilizar todas las observaciones individuales disponibles con el objetivo de eliminar el sesgo de agregación, preferiblemente utilizando esas observacioes para realizar un solo análisis de regresión. Esta es la metodología de preferencia que se utilizará a lo largo de la presente investigación. Adicionalmente, resulta útil emplear toda la información disponible a efectos de realizar pruebas de hipótesis. Un ejercicio de este tipo es mucho más poderoso en cuanto a la precisión de las pruebas, cuando se utilizan $T \cdot N$ observaciones en vez de utilizar solamente las $T$ disponibles cuando se obtiene un promedio para cada mes.

Para todas las estimaciones realizadas se ejecuta primero una prueba de Wooldridge ${ }^{10}$ para verificar la existencia de autocorrelación, las cuales resultan en el rechazo de la hipótesis nula de no autocorrelación ${ }^{11}$. Por lo tanto, es importante aclarar que serán reportados los errores estándar de NeweyWest. Esto permite corregir (cuando es requerido hacerlo) por autocorrelación en datos de panel, y se elige hacerlo para un periodo máximo de un año, tal y como se realiza en Mankiw et al. (2004).

\section{Hipótesis de racionalidad}

El sesgo que contienen las expectativas de inflación es evidente gráficamente y es sencillo de verificar empíricamente. Siguiendo la metodología para someter a prueba la hipótesis de sesgo en los pronósticos utilizada por Mankiw et al. (2004), se emplea la regresión que se detalla a continuación.

$$
\pi_{t}-E_{i, t-12} \pi_{t}=\alpha
$$

Tal y como describen los autores, un ejercicio de este tipo es equivalente a correr una regresión de Mínimos Cuadrados Ordinarios (MCO) de la forma $\pi_{t}=a+b * E_{i, t-12} \pi_{t}$, en la cual una prueba de hipótesis de racionalidad sería una prueba conjunta de $a=0$ y $b=1$. De la forma que se plantea en la ecuación 5, la prueba consiste en evaluar si el coeficiente es significativamente distinto de cero. Si los pronósticos fueran eficientes, no se debería poder predecir los errores que se generan a partir de los mismos. El resultado de la regresión en el cuadro 1 indica que es posible predecir el error, lo que hace rechazar la hipótesis más sencilla de racionalidad. A lo largo de la muestra existe un sesgo de las expectativas de casi 1,6 puntos porcentuales por encima de los datos reales de inflación ${ }^{12}$.

$10 \quad$ La prueba de autocorrelación de Wooldridge es utilizada usualmente para analizar los errores de modelos lineales de datos de panel. Para mayor detalle, ver Wooldridge (2002).

11 Diebold y Lopez (1995) consideran que para que los pronósticos sean óptimos debe cumplirse que: 1. Los errores tengan media cero; 2 . Los errores de un periodo adelante sean ruido blanco; 3 . Los pronósticos $s$ periodos adelante del periodo actual $k$ sean, a lo sumo $\mathrm{MA}(k-s)$ y 4 . La varianza de los errores sea no decreciente en el horizonte de pronóstico.

12 En el cuadro 1 y el cuadro 2 no se detalla información relativa al estadístico $\mathrm{R}^{2}$, debido a que cuando se omiten variables explicativas este toma un valor de cero. 
CUADRO 1

RESULTADO DE MCO DE ECUACIÓN (5)

DICIEMBRE 2006 - ABRIL 2013

\begin{tabular}{cccc}
\hline Variable & Coeficiente & (Error Est. N-W) & N \\
\hline Constante $(\alpha)$ & $-1,562$ & $(0,145)$ & 2702 \\
\hline
\end{tabular}

Nivel de significancia : †: $10 \% *: 5 \% * *: 1 \%$

No obstante, este sesgo es diferente no solo en magnitud sino en signo, cuando se evalúan los dos subperiodos separados por el cambio estructural identificado por Torres (2012). Para el primer subperiodo comprendido entre diciembre de 2006 y abril de 2009, las expectativas subestiman el resultado de la inflación, mientras que en el segundo subperiodo, que abarca de mayo 2009 a abril de 2013, el sesgo es hacia una sobreestimación de la inflación. En el resto del documento se identificará el periodo previo al quiebre estructural con un subíndice 0, mientras que 1 representará el periodo posterior al quiebre estructural. En el cuadro 2 se identifica el mismo coeficiente de sesgo calculado para la regresión correspondiente a la ecuación (5) para los dos periodos.

CUADRO 2

RESULTADO DE MCO DE ECUACIÓN (5) PARA DOS PERÍODOS

\begin{tabular}{cccc}
\hline Variable & Coeficiente & (Error Est. N-W) & N \\
\hline Constante $\left(\alpha_{0}\right)$ & 0,946 & $(0,351)$ & 685 \\
Constante $\left(\alpha_{1}\right)$ & $-2,414$ & $(0,119)$ & 2017 \\
\hline
\end{tabular}

Nivel de significancia : † : $10 \% *: 5 \% * *: 1 \%$

Fuente: Elaboración propia.

Uno de los primeros análisis que es interesante realizar es identificar si todos los estratos tienen sesgos similares, o si es más bien el conjunto de individuos pertenecientes a uno de los estratos consultados el que ocasiona el sesgo presente en el agregado. El gráfico 3 presenta las series de expectativas de inflación para el mismo periodo agregadas y también desagregadas por estrato. Es notorio resaltar que, a pesar de haber una mayor volatilidad en algunos de los estratos (debida principalmente al reducido número de individuos consultados), las cuatro series de datos desagregados reflejan comportamientos muy similares.

\section{GRÁFICO 3}

EXPECTATIVAS DE INFLACIÓN AGREGADAS Y POR ESTRATO

ENERO 2007 - ABRIL 2013

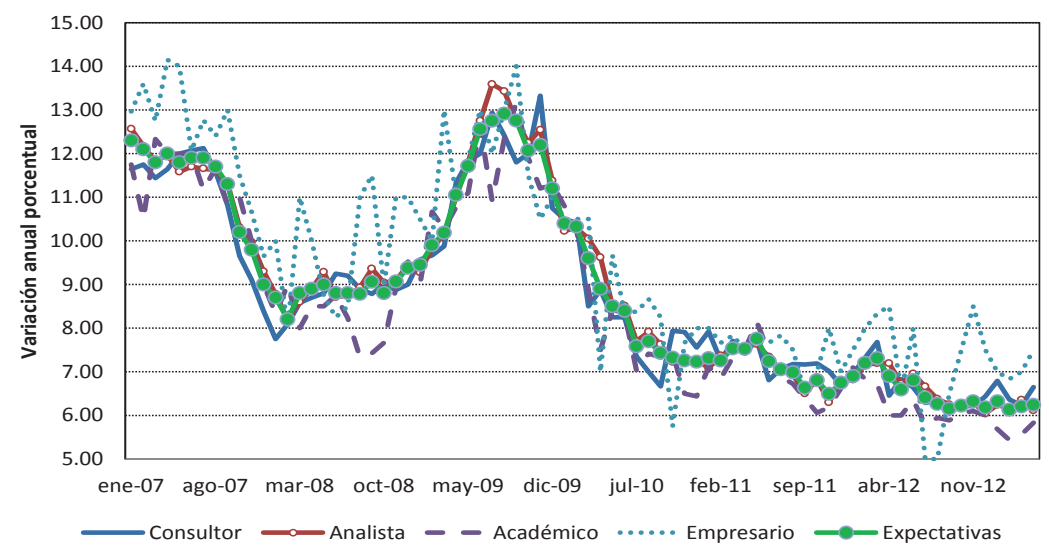

Fuente: Elaboración propia. 
Sin embargo, esta forma de visualizar la información implica ignorar el sesgo de agregación que se discutió previamente. Una forma mucho más apropiada de visualizar los datos es la que se presenta en el gráfico 4, en el cual las observaciones individuales se agrupan con marcadores que varían en tamaño dependiendo de la cantidad de respuestas coincidentes. Esta presentación de la información permite identificar casos, en particular para los consultores y analistas bursátiles, en los cuales algunos pronósticos individuales se alejan significativamente del promedio y no se corrigen rápidamente, a pesar de estar considerablemente lejos del promedio. Además, los pronósticos individuales alrededor de la serie agregada no parecen reflejar una distribución normal, en particular para el periodo posterior al quiebre estructural ${ }^{13}$.

\section{GRÁFICO 4 \\ EXPECTATIVAS DE INFLACIÓN DESAGREGADAS POR ESTRATO DATOS RECOPILADOS DE ENERO 2006 A ABRIL 2013}
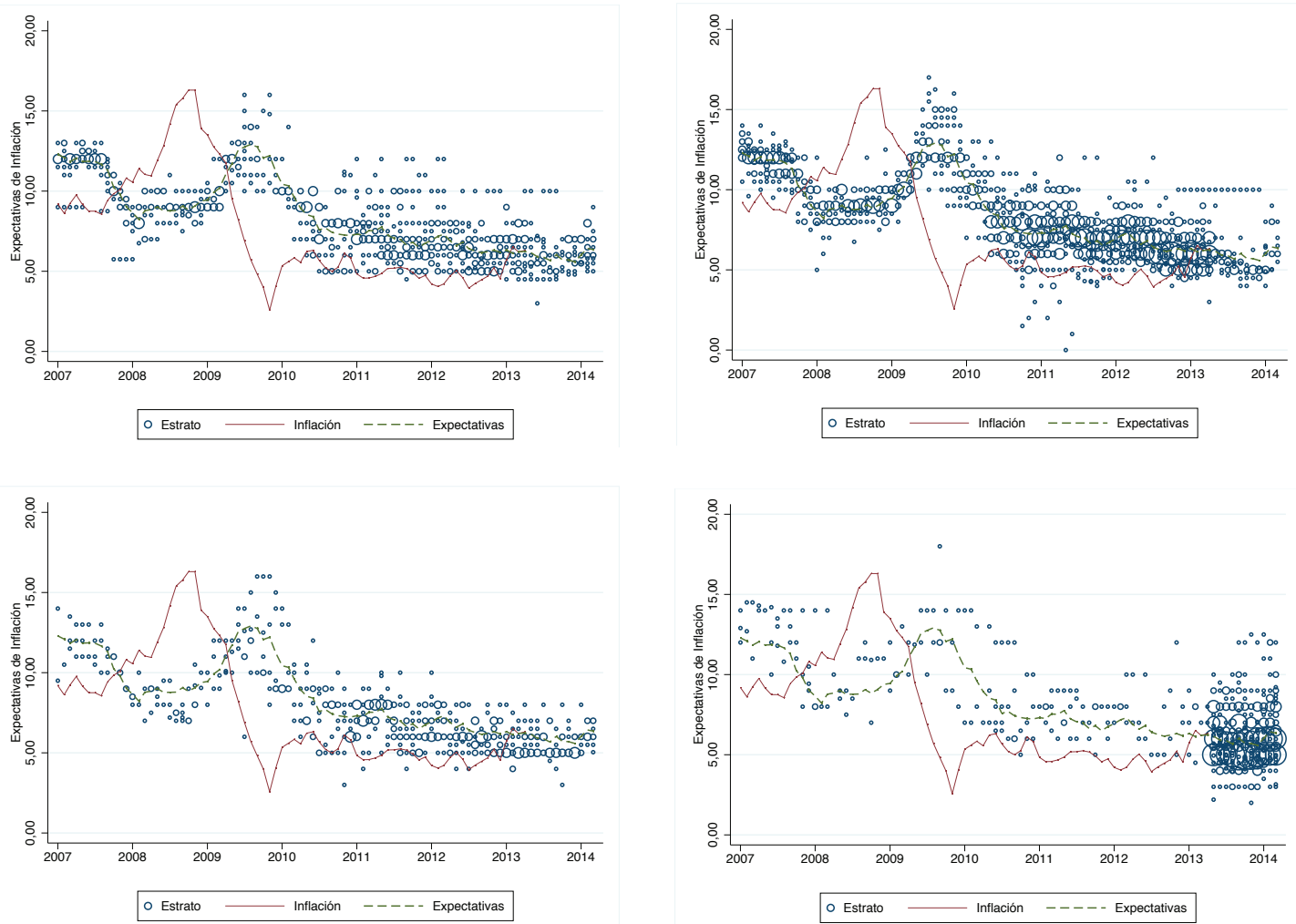

Fuente: Elaboración propia.

El cuadro 3 confirma que existen sesgos significativos en todos los estratos de individuos, aunque algunos son mucho más pronunciados que otros. Con excepción de los empresarios, antes del quiebre estructural, todos los análisis muestran sesgos significativos en la misma dirección que el dato para toda la población: una subestimación de la inflación previo al quiebre, y una sobreestimación posterior al mismo ${ }^{14}$. Resulta interesante destacar que previo a abril de 2009 no existía

13 En el Anexo A se pueden apreciar los datos recientes para todos los estratos en un solo gráfico.

14 Aunque, como muestra el cuadro, para los tres estratos restantes y en el periodo previo al quiebre estructural, el coeficiente es significativo con un $10 \%$ de confianza. 
un sesgo por parte de los empresarios que fuera estadísticamente significativo. Sin embargo, posterior a mayo de 2009, los empresarios constituyen el grupo en el que los datos reflejan un sesgo de mayor magnitud.

Mankiw et al. (2004) realizan un análisis similar para la economía estadounidense y encuentran que solamente en una de las cuatro encuestas analizadas existe un sesgo estadísticamente significativo. El caso costarricense es contrastante dada su dimensión. En el periodo posterior al quiebre estructural este sesgo alcanza niveles que hacen a la expectativa casi duplicar el nivel de la inflación que se hace efectiva.

\section{CUADRO 3}

RESULTADO DE MCO DE ECUACIÓN (5) PARA CUATRO ESTRATOS

\begin{tabular}{|c|c|c|c|c|}
\hline Variable & Coeficiente & $\begin{array}{c}\text { (Error Est. } \\
\mathrm{N}-\mathrm{W})\end{array}$ & $\mathrm{N}$ & $\mathrm{R}^{2}$ \\
\hline \multicolumn{5}{|l|}{ Muestra completa } \\
\hline Constante $\left(\alpha_{\text {Consultores }}\right)$ & $-1,373$ & $(0,294)$ & 670 & 0 \\
\hline Constante $\left(\alpha_{\text {Anal. Burs. }}\right)$ & $-1,611$ & $(0,195)$ & 1443 & 0 \\
\hline Constante ( $\left.\alpha_{\text {Académicos }}\right)$ & $-1,426$ & $(0,363)$ & 421 & 0 \\
\hline Constante $\left(\alpha_{\text {Empresarios }}\right)$ & $-2,236$ & $(0,599)$ & 168 & 0 \\
\hline \multicolumn{5}{|l|}{ Anterior a quiebre estructural } \\
\hline Constante $\left(\alpha_{0, \text { Consultores }}\right)$ & 1,121 & $(0,631)$ & 198 & 0 \\
\hline Constante $\left(\alpha_{0, \text { Anal. Burs. }}\right)$ & 0,849 & $(0,487)$ & 353 & 0 \\
\hline Constante $\left(\alpha_{0, \text { Académicos }}\right)$ & 1,923 & $(1,095)$ & 76 & 0 \\
\hline Constante $\left(\alpha_{0, \text { Empresarios }}\right)$ & $-0,337$ & $(1,189)$ & 58 & 0 \\
\hline \multicolumn{5}{|l|}{ Posterior a quiebre estructural } \\
\hline Constante $\left(\alpha_{1, \text { Consultores }}\right)$ & $-2,419$ & $(0,234)$ & 472 & 0 \\
\hline Constante $\left(\alpha_{1, \text { Anal. Burs. }}\right)$ & $-2,408$ & $(0,161)$ & 1090 & 0 \\
\hline Constante $\left(\alpha_{1, \text { Anal. Burs. }}\right)$ & $-2,163$ & $(0,286)$ & 345 & 0 \\
\hline Constante $\left(\alpha_{1, \text { Empresarios }}\right)$ & $-3,238$ & $(0,559)$ & 110 & 0 \\
\hline
\end{tabular}

Nivel de significancia : † : $10 \% *: 5 \% * *: 1 \%$

Fuente: Elaboración propia.

Al confrontar estos resultados, la pregunta que debe saltar a la mente es si estos sesgos son significativamente diferentes el uno del otro. En la Ecuación (6) se adicionan tres variables dicotómicas con respecto a la Ecuación (5) que representan los estratos de analistas bursátiles (ab), académicos (ac) y empresarios (em). Los resultados de esta regresión para todo 
el periodo se presentan en el cuadro 4, y son sumamente similares a los correspondientes del periodo posterior al quiebre estructural.

$$
\pi_{t}-E_{t-12} \pi_{t}=\alpha+\alpha_{a b}+\alpha_{a c}+\alpha_{e m}
$$

Ningún sesgo es significativamente diferente al de los consultores (el estrato que se utiliza como base para comparación). Esto ocurre tanto en la muestra total (resultado mostrado en el cuadro) como por subperiodos. Al realizar una serie de pruebas de hipótesis $F$, no es posible rechazar para todos los periodos considerados, que todos los coeficientes de las variables introducidas sean iguales a cero.

CUADRO 4

RESULTADO DE MCO DE ECUACIÓN (6)

DICIEMBRE 2006 - ABRIL 2013

\begin{tabular}{ccccc}
\hline Variable & Coeficiente & (Error Est. N-W) & $\mathrm{N}$ & $\mathrm{R}^{2}$ \\
\hline$\alpha_{a b}$ & $-0,238$ & $(0,353)$ & 2702 & 0,004 \\
$\alpha_{a c}$ & $-0,053$ & $(0,467)$ & & \\
$\alpha_{e m}$ & $-0,863$ & $(0,665)$ & & \\
$\alpha$ & $-1,373$ & $(0,294)$ & \\
\hline
\end{tabular}

Nivel de significancia : †: $10 \% *: 5 \% * *: 1 \%$

Fuente: Elaboración propia.

Es importante destacar nuevamente la virtud de realizar las modificaciones a las pruebas de hipótesis por la presencia de autocorrelación en datos de panel. Además, es relevante que sean utilizados los datos de expectativas de manera individual para que los sesgos de agregación sean eliminados y las pruebas de hipótesis tengan una mayor potencia al usar una cantidad significativamente mayor de datos. Si no se tomara en cuenta la autocorrelación en los datos, sería posible rechazar las hipótesis de que los sesgos por estrato son iguales a cero y equivalentes entre sí, lo cual confirma la importancia de realizar el análisis de la manera en que ha sido propuesto en esta investigación.

Dado que la encuesta de expectativas permite darle seguimiento a individuos a través del tiempo, es posible realizar el mismo análisis de sesgo separadamente para cada uno de los agentes consultados. Los resultados son sumamente similares, pero pueden destacarse algunas particularidades. Existen 64 agentes, para los cuales se cuenta con la suficiente cantidad de observaciones para realizar el ajuste por autocorrelación para un año completo (de 108 individuos originalmente), de manera que sea posible realizar el análisis de regresión. De estos, ningún agente presenta un sesgo estadísticamente significativo que subestime el resultado de inflación. Para 30 agentes no se puede rechazar la hipótesis nula de que el sesgo ( ) es igual a cero. Sin embargo, para el 57\% de los agentes (34 individuos) existe un sesgo hacia la sobreestimación de la inflación.

Cuando la misma desagregación por individuos se realiza para el periodo más reciente (posterior al quiebre estructural), los resultados son aún más contundentes. De 58 agentes con información suficiente, nuevamente ninguno presenta un sesgo hacia la subestimación de la inflación, mientras que en solamente seis individuos no existe un sesgo estadísticamente significativo. Para 
el resto de los casos (52, o el 90\%), hay un sesgo relevante hacia la sobreestimación del resultado de la variación interanual del IPC. Para algunos individuos consultados, el sesgo es sumamente importante. Existen varios casos en los que el mismo supera cinco puntos porcentuales. Dados los resultados de inflación de los años recientes, esto significa que este individuo, un año antes, espera que la inflación tome un valor superior en más de un $100 \%$ de la que en realidad ocurre.

Otra hipótesis con respecto a la racionalidad de los agentes económicos que se puede someter a prueba es cuán útil es el dato de la expectativa de inflación para predecir el error que esos datos tendrán cuando se reconozca la variación del IPC. Si no existieran sesgos en los pronósticos, la predicción que se realiza en determinado periodo no debería tener utilidad alguna para predecir los errores que serán causados por dicha expectativa. La Ecuación (7) permite realizar este análisis utilizando MCO.

$$
\pi_{t}-E_{t-12} \pi_{t}=\alpha+\beta E_{t-12} \pi_{t}
$$

El cuadro 5 presenta los datos analizados por estrato utilizando la totalidad de la muestra, el periodo antes del quiebre estructural y el periodo posterior. Los coeficientes $\alpha$ y $\beta$ son altamente significativos en todos los casos. Las hipótesis nulas en las que se evalúa conjuntamente $\alpha=0$ y $\beta=0$ se rechazan en todas las ocasiones con un nivel de confianza del 1\%. Los resultados son similares cuando se consideran los datos agrupados sin considerar el estrato al que pertenecen las personas.

CUADRO 5

RESULTADO DE MCO DE ECUACIÓN (7) PARA CUATRO ESTRATOS

\begin{tabular}{|c|c|c|c|c|}
\hline Variable & Coeficiente & (Error Est. N-W) & $\mathrm{N}$ & $\mathrm{R}^{2}$ \\
\hline \multicolumn{5}{|l|}{ Muestra completa } \\
\hline Constante $\left(\alpha_{\text {Consultores }}\right)$ & 2,618 & $(0,615)$ & 670 & 0,132 \\
\hline$E_{t-12^{\pi} t}\left(\beta_{\text {Consultores }}\right)$ & $-0,479$ & $(0,081)$ & & \\
\hline Constante $\left(\alpha_{\text {Anal. Burs. }}\right)$ & 2,586 & $(0,413)$ & 1443 & 0,168 \\
\hline$E_{t-12} \Pi_{t}\left(\beta_{\text {Anal. Burs. }}\right)$ & $-0,509$ & $(0,059)$ & & \\
\hline Constante $\left(\alpha_{\text {Académicos }}\right)$ & 3,381 & $(0,775)$ & 421 & 0,225 \\
\hline$E_{t-12{ }^{\prime} t}\left(\beta_{\text {Académicos }}\right)$ & $-0,621$ & $(0,115)$ & & \\
\hline Constante $\left(\alpha_{\text {Empresarios }}\right)$ & 3,337 & $(1,172)$ & 168 & 0,242 \\
\hline$E_{t-12^{\pi} t}\left(\beta_{\text {Empresarios }}\right)$ & $-0,589$ & $(0,126)$ & & \\
\hline \multicolumn{5}{|l|}{ Anterior a quiebre estructural } \\
\hline Constante $\left(\alpha_{0, \text { Consultores }}\right)$ & 18,837 & $(2,011)$ & 198 & 0,690 \\
\hline$E_{t-12} \Pi_{t}\left(\beta_{0, \text { consultores }}\right)$ & $-1,750$ & $(0,177)$ & & \\
\hline Constante $\left(\alpha_{0, \text { Anal. Burs. }}\right)$ & 20,678 & $(1,186)$ & 353 & 0,738 \\
\hline
\end{tabular}




\begin{tabular}{|c|c|c|c|c|}
\hline Variable & Coeficiente & (Error Est. N-W) & $\mathrm{N}$ & $\mathrm{R}^{2}$ \\
\hline$E_{t-12}{ }^{\pi}\left(\beta_{0, \text { Anal. Burs. }}\right)$ & $-1,906$ & $(0,100)$ & & \\
\hline Constante $\left(\alpha_{0, \text { Académicos }}\right)$ & 20,664 & $(1,298)$ & 76 & 0,783 \\
\hline$E_{t-12^{\Pi} t}\left(\beta_{0, \text { Académicos }}\right)$ & $-1,905$ & $(0,120)$ & & \\
\hline Constante $\left(\alpha_{0, \text { Empresarios }}\right)$ & 16,453 & $(1,525)$ & 58 & 0,718 \\
\hline$E_{t-12^{\Pi} t}\left(\beta_{0, \text { Empresarios }}\right)$ & $-1,480$ & $(0,110)$ & & \\
\hline \multicolumn{5}{|l|}{ Posterior a quiebre estructural } \\
\hline Constante $\left(\alpha_{1, \text { Consultores }}\right)$ & 4,263 & $(0,259)$ & 472 & 0,810 \\
\hline$E_{t-12} \Pi_{t}\left(\beta_{1, \text { Consultores }}\right)$ & $-0,880$ & $(0,036)$ & & \\
\hline Constante $\left(\alpha_{1, \text { Anal. Burs. }}\right)$ & 4,565 & $(0,168)$ & 1090 & 0,831 \\
\hline$E_{t-12} \Pi_{t}\left(\beta_{1, \text { Anal. Burs. }}\right)$ & $-0,924$ & $(0,023)$ & & \\
\hline Constante $\left(\alpha_{1, \text { Académicos }}\right)$ & 4,741 & $(0,260)$ & 345 & 0,841 \\
\hline$E_{t-12} \Pi_{t}\left(\beta_{1, \text { Académicos }}\right)$ & $-0,949$ & $(0,035)$ & & \\
\hline Constante $\left(\alpha_{1, \text { Empresarios }}\right)$ & 4,710 & $(0,333)$ & 110 & 0,866 \\
\hline$E_{t-12}{ }_{t}\left(\beta_{1, \text { Empresarios }}\right)$ & $-0,938$ & $(0,037)$ & & \\
\hline
\end{tabular}

Nivel de significancia : †: $10 \% *: 5 \% * *: 1 \%$

Fuente: Elaboración propia.

Estos resultados confirman que es posible predecir la magnitud de los errores del pronóstico basándose en las expectativas reveladas por los agentes consultados. Por ejemplo, para el subperiodo posterior al quiebre estructural, cada punto porcentual adicional en las expectativas de inflación implica un error con respecto a la inflación efectiva, cercano al 1 punto porcentual (p.p.). En el caso específico de un individuo que haya externado que la inflación vaya a ser $8 \%$, posiblemente va a errar su pronóstico por 4 p.p. En el Anexo B se presenta un gráfico con la relación entre el error y las expectativas manifestadas por los agentes.

Una prueba adicional que se puede realizar con respecto a la racionalidad de las expectativas es evaluar la persistencia de los errores. Bajo la hipótesis de racionalidad de las expectativas, los errores pasados contendrían información que contribuya a reducir los errores del futuro. Si fuese cierto que los individuos manifiestan expectativas racionales, sería de esperar que, al obtener cada mes información con respecto a la inflación efectiva para ese periodo, y consecuentemente para el error que tuvo su predicción un año atrás, los errores se corrijan para las predicciones que se hagan hacia el futuro. Si por el contrario, esta información no es explotada al momento de generar una nueva expectativa, la hipótesis de racionalidad podría ser cuestionada. Se estaría en un proceso de ajuste sumamente lento, ya que se ignoran los errores pasados como fuente de aprendizaje en el presente. La ecuación (8) representa ese ejercicio. La persistencia de los errores es medida por el coeficiente $\beta$ para cada uno de los estratos.

$$
\pi_{t}-E_{t-12} \pi_{t}=\alpha+\beta\left(\pi_{t-12}-E_{t-24} \pi_{t-12}\right)
$$


En este caso la cantidad de información disponible para realizar las estimaciones disminuye significativamente, ya que para ser considerado en este ejercicio, un individuo debe estar presente en la muestra para el mismo mes en dos años consecutivos. Es usual que algunos individuos no contesten en algunos meses la consulta o que salgan de la muestra por alguna razón, por lo que la cantidad de observaciones utilizables cae de manera importante. No obstante, esta forma de utilizar los datos tiene la virtud de identificar la información que tiene cada persona de sus propios errores pasados al momento de realizar nuevos pronósticos, por lo que los coeficientes tienen la interpretación correcta sin riesgo de implicar un sesgo de agregación.

El cuadro 6 presenta los resultados de las regresiones respectivas por estrato. Es posible apreciar que cuando se analiza todo el periodo de la muestra hay persistencia de los errores en los estratos de analistas bursátiles y académicos. Esta persistencia no está presente en ambas partes de la muestra, solamente después del quiebre estructural, e incluye al estrato de consultores ${ }^{15}$.

CUADRO 6

RESULTADO DE MCO DE ECUACIÓN (8) PARA CUATRO ESTRATOS

\begin{tabular}{|c|c|c|c|c|}
\hline Variable & Coeficiente & $\begin{array}{l}\text { (Error Est. } \\
\quad \mathrm{N}-\mathrm{W})\end{array}$ & $\mathrm{N}$ & $\mathrm{R}^{2}$ \\
\hline \multicolumn{5}{|l|}{ Muestra completa } \\
\hline Constante $\left(\alpha_{\text {Consultores }}\right)$ & $-1,584$ & $(0,462)$ & 403 & 0,024 \\
\hline$\pi_{t-12}-E_{t-24} \pi_{t-12}\left(\beta_{\text {Consultores }}\right)$ & $-0,164$ & $(0,117)$ & & \\
\hline Constante $\left(\alpha_{\text {Anal. Burs. }}\right)$ & $-2,166$ & $(0,313)$ & 783 & 0,098 \\
\hline$\pi_{t-12}-E_{t-24} \pi_{t-12}\left(\beta_{\text {Anal. Burs. }}\right)$ & $-0,293$ & $(0,069)$ & & \\
\hline Constante ( $\left.\alpha_{\text {Académicos }}\right)$ & $-1,850$ & $(0,522))$ & 270 & 0,121 \\
\hline$\pi_{t-12}-E_{t-24} \pi_{t-12}\left(\beta_{\text {Académicos }}\right)$ & $-0,294$ & $(0,117)$ & & \\
\hline Constante $\left(\alpha_{\text {Empresarios }}\right)$ & $-2,457$ & $(0,778)$ & 86 & 0,028 \\
\hline$\pi_{t-12}-E_{t-24} \pi_{t-12}\left(\beta_{\text {Empresarios }}\right)$ & $-0,141$ & $(0,147)$ & & \\
\hline \multicolumn{5}{|l|}{ Anterior a quiebre estructural } \\
\hline Constante $\left(\alpha_{0, \text { Consultores }}\right)$ & 3,834 & $(0,291)$ & 73 & 0,004 \\
\hline$\pi_{t-12}-E_{t-24} \pi_{t-12}\left(\beta_{0, \text { Consultores }}\right)$ & $-0,050$ & $(0,105)$ & & \\
\hline Constante $\left(\alpha_{0, \text { Anal. Burs. }}\right)$ & 3,717 & $(0,275)$ & 117 & 0,009 \\
\hline$\pi_{t-12}-E_{t-24} \pi_{t-12}\left(\beta_{0, \text { Anal. Burs. }}\right)$ & $-0,081$ & $(0,086)$ & & \\
\hline Constante $\left(\alpha_{0, \text { Académicos }}\right)$ & 3,958 & $(0,628)$ & 28 & 0,032 \\
\hline$\pi_{t-12}-E_{t-24} \pi_{t-12}\left(\beta_{0, \text { Académicos }}\right)$ & $-0,186$ & $(0,250)$ & & \\
\hline Constante $\left(\alpha_{0, \text { Empresarios }}\right)$ & 2,157 & $(0,556)$ & 15 & 0,034 \\
\hline $\begin{array}{l}\pi_{t-12}-E_{t-24} \pi_{t-12} \\
\left(\beta_{0, \text { Empresarios }}\right)\end{array}$ & 0,171 & $(0,237)$ & & \\
\hline
\end{tabular}

Continúa...

15 Si se realiza el mismo ejercicio utilizando solamente datos de aquellos individuos que han estado en la muestra por un periodo extendido de tiempo (observaciones para al menos 36 meses), los resultados son casi idénticos. 


\begin{tabular}{lcccc}
\hline Variable & Coeficiente & $\begin{array}{c}\text { (Error Est. } \\
\text { N-W })\end{array}$ & N & $\mathbf{R}^{2}$ \\
\hline Posterior a quiebre estructural & & & & \\
\hline Constante $\left(\alpha_{1, \text { consultores }}\right)$ & $-2,930$ & $(0,326)$ & 330 & 0,148 \\
$\pi_{t-12}-E_{t-24} \pi_{t-12}\left(\beta_{1, \text { Consultores }}\right)$ & $-0,266$ & $(0,090)$ & & \\
Constante $\left(\alpha_{1, \text { Anal. Burs. })}\right.$ & $-3,236$ & $(0,209)$ & 666 & 0,288 \\
$\pi_{t-12}-E_{t-24} \pi_{t-12}\left(\beta_{1, \text { Anal. Burs. }}\right)$ & $-0,342$ & $(0,054)$ & & \\
Constante $\left(\alpha_{1, \text { Académicos }}\right)$ & $-2,587$ & $(0,410)$ & 242 & 0,274 \\
$\pi_{t-12}-E_{t-24} \pi_{t-12}\left(\beta_{1, \text { Académicos }}\right)$ & $-0,339$ & $(0,105)$ & & \\
Constante $\left(\alpha_{1, \text { Empresarios }}\right)$ & $-3,452$ & $(0,756)$ & 71 & \\
$\pi_{t-12}-E_{t-24} \pi_{t-12}$ & $-0,203$ & $(0,123)$ & & \\
$\left(\beta_{1, \text { Empresarios }}\right)$ & & & & \\
Nivel de significancia $: \dagger: 10 \% *: 5 \% * *: 1 \%$ & & & \\
\hline
\end{tabular}

Fuente: Elaboración propia.

En este segundo periodo existe persistencia en los errores que varía del 26\% al 34\% para un año posterior. Mankiw et al. (2004) encontraron una persistencia significativa de los errores de alrededor de un 50\% para EUA. Para los empresarios no existe evidencia estadística de persistencia, lo que indicaría que este grupo aprende de los errores de manera más rápida que el resto de los estratos ${ }^{16}$.

El periodo inflacionario de 2008-2009 tuvo como uno de sus efectos destacables la generación de esa persistencia de alrededor de un $30 \%$ para la mayoría los estratos. Es relevante identificar si el grado de persistencia de los diferentes estratos es estadísticamente diferente el uno de otro. Para verificar esto se realizó una prueba de hipótesis con la especificación de la Ecuación (9).

$$
\begin{gathered}
\pi_{t}-E_{t-12} \pi_{t}=\alpha+\beta_{c o}\left(\pi_{t-12}-E_{t-24} \pi_{t-12}\right)+\beta_{a b}\left(\pi_{t-12}-E_{t-24} \pi_{t-12}\right)+ \\
\beta_{a c}\left(\pi_{t-12}-E_{t-24} \pi_{t-12}\right)+\beta_{e m}\left(\pi_{t-12}-E_{t-24} \pi_{t-12}\right)
\end{gathered}
$$

Para el periodo previo al quiebre estructural no es posible descartar estadísticamente que todos los coeficientes $\beta_{i}=0$. Para el periodo posterior a la crisis esta misma hipótesis nula se rechaza. Sin embargo, los coeficientes para cada estrato no son estadísticamente distintos entre sí, por lo que no es posible rechazar la hipótesis nula $\beta_{c o}=\beta_{a b}=\beta_{a c}=\beta_{e m}$. No obstante, dados los resultados mostrados en el cuadro 7 , es posible presumir que con algo más de información ${ }^{17}$, para el estrato de empresarios eventualmente se confirme que la menor persistencia sea estadísticamente diferente del resto.

$\overline{16}$ Si se utiliza un quiebre estructural 12 meses adelante (momento a partir del cual el promedio de las expectativas toma valores de un dígito) se encuentran resultados ligeramente diferentes. Hay persistencia del error en el estrato de consultores para ambos periodos, pero con signo contrario. También es destacable que para los demás estratos la persistencia desaparece posterior al quiebre estructural.

17 Por ejemplo, para la regresión utilizando datos de este estrato en el periodo posterior al quiebre estructural hay apenas 71 observaciones que se pueden utilizar, mientras que para los demás estratos hay de 3 a 10 veces más observaciones. 
CUADRO 7

RESULTADO DE MCO DE ECUACIÓN (9)

MAYO 2009 - ABRIL 2013

\begin{tabular}{ccccc}
\hline Variable & Coeficiente & (Error Est. N-W) & $\mathbf{N}$ & $\mathbf{R}^{2}$ \\
\hline$\beta_{c o}$ & $-0,280$ & $(0,076)$ & 1309 & 0,236 \\
$\beta_{a b}$ & $-0,321$ & $(0,053)$ & & \\
$\beta_{a c}$ & $-0,385$ & $(0,078)$ & & \\
$\beta_{e m}$ & $-0,159$ & $(0,106)$ & & \\
$\alpha$ & $-3,045$ & $(0,164)$ & & \\
\hline
\end{tabular}

Nivel de significancia : †: $10 \% *: 5 \% * *: 1 \%$

Fuente: Elaboración propia.

Adicionalmente, en el cuadro 4 se mostraba que, aunque tampoco es estadísticamente significativo, el sesgo de los empresarios parece ser el mayor, y el resultado es válido también para el segundo periodo. Esto podría indicar que este grupo de individuos sea el que inicialmente comete mayores errores en sus predicciones, pero que eventualmente los corrige de manera más veloz. El cuadro 7 indica que esta persistencia de los errores de empresarios es menor a un año. Esto implicaría que en la actualización de la muestra discutida previamente (que se utiliza a partir de julio 2012), es menos probable que los errores se mantengan por un periodo extendido de tiempo.

Una última hipótesis que es relevante analizar es si los errores de pronóstico podrían haber sido menores si los agentes hubieran incorporado alguna información macroeconómica que estuviera disponible en el momento en que manifestaron la expectativa. En la ecuación (10) se incorpora información de la inflación, nivel de desempleo $(U)^{18}$ y tasa de interés $(i)^{19}$, para el mes inmediatamente previo al momento en el que se realiza la consulta. En el caso que dichas variables sean estadísticamente significativas, se podría inferir que estas ayudan a explicar el comportamiento de los errores, por lo que los mismos se hubieran podido reducir si los agentes encuestados los hubieran tomado en cuenta a la hora de formular sus expectativas. En el Cuadro 8 se presentan los resultados para el período posterior al quiebre estructural ${ }^{20}$.

$$
\pi_{t}-E_{t-12} \pi_{t}=\alpha+\beta E_{t-12} \pi_{t}+\gamma \pi_{t-13}+\mathrm{ki} i_{t-13}+\delta U_{t-13}
$$

18 Esta variable se construye utilizando los datos de la nueva Encuesta Continua de Empleo publicada a partir de agosto de 2010, y para los meses previos se extrapolan los datos anuales de la Encuesta Nacional de Hogares (ENAHO). El nivel de la serie es provisto por un promedio de ambas encuestas. A pesar de que los agentes no conocían el dato de desempleo trimestral (dado que la encuesta fue hecha pública recientemente), se utiliza este dato como una variable de aproximación hacia lo que los agentes perciben en la economía al momento de realizar el pronóstico.

19 Se utiliza la Tasa Básica Pasiva. Alternativamente se utilizó la tasa promedio de depósitos del sistema financiero, con resultados similares.

20 Este periodo es el que permite utilizar datos más precisos gracias a la metodología que se utilizó para construir la serie de desempleo. 
CUADRO 8

RESULTADO DE MCO DE ECUACIÓN (10) POR ESTRATOS

\begin{tabular}{|c|c|c|c|c|}
\hline Variable & Coeficiente & (Error Est. N-W) & $\mathrm{N}$ & $\mathrm{R}^{2}$ \\
\hline \multicolumn{5}{|c|}{ Posterior a quiebre estructural - estratos } \\
\hline Constante $\left(\alpha_{\text {Consultores }}\right)$ & 10,896 & $(1,826)$ & 439 & 0,829 \\
\hline$E_{t-12} \pi_{t}\left(\beta_{\text {Consultores }}\right)$ & $-0,972$ & $(0,039)$ & & \\
\hline$\pi_{t-13}\left(\gamma_{\text {consultores }}\right)$ & $-0,181$ & $(0,050)$ & & \\
\hline$i_{t-13}\left(\kappa_{\text {Consultores }}\right)$ & 0,244 & $(0,031)$ & & \\
\hline$U_{t-13}\left(\delta_{\text {Consultores }}\right)$ & $-0,929$ & $(0,207)$ & & \\
\hline Constante $\left(\alpha_{\text {Anal. Burs. }}\right)$ & 9,722 & $(1,419)$ & 971 & 0,846 \\
\hline$E_{t-12} \pi_{t}\left(\beta_{\text {Anal. Burs. }}\right)$ & $-0,940$ & $(0,030)$ & & \\
\hline$\pi_{t-13}\left(\gamma_{\text {Anal. Burs. }}\right)$ & $-0,198$ & $(0,039)$ & & \\
\hline$i_{t-13}\left(\kappa_{\text {Anal. Burs. }}\right)$ & 0,228 & $(0,022)$ & & \\
\hline$U_{t-13}\left(\delta_{\text {Anal. Burs. }}\right)$ & $-0,773$ & $(0,167)$ & & \\
\hline Constante ( $\left.\alpha_{\text {Académicos }}\right)$ & 9,854 & $(2,576)$ & 320 & 0,856 \\
\hline$E_{t-12} \pi_{t}\left(\beta_{\text {Académicos }}\right)$ & $-1,001$ & $(0,047)$ & & \\
\hline$\pi_{t-13}\left(\gamma_{\text {Académicos }}\right)$ & $-0,171$ & $(0,062)$ & & \\
\hline$i_{t-13}\left(\kappa_{\text {Académicos }}\right)$ & 0,235 & $(0,040)$ & & \\
\hline$U_{t-13}\left(\delta_{\text {Académicos }}\right)$ & $-0,762$ & $(0,302)$ & & \\
\hline Constante $\left(\alpha_{\text {Empresarios }}\right)$ & 7,288 & $(3,472)$ & 101 & 0,884 \\
\hline$E_{t-12} \pi_{t}\left(\beta_{\text {Empresarios }}\right)$ & $-0,933$ & $(0,043)$ & & \\
\hline$\pi_{t-13}\left(\gamma_{\text {Empresarios }}\right)$ & $-0,133$ & $(0,103)$ & & \\
\hline$i_{t-13}\left(\kappa_{\text {Empresarios }}\right)$ & 0,131 & $(0,076)$ & & \\
\hline$U_{t-13}\left(\delta_{\text {Empresarios }}\right)$ & $-0,394$ & $(0,439)$ & & \\
\hline
\end{tabular}

Nivel de significancia $: \dagger: 10 \% *: 5 \% * *: 1 \%$

Fuente: Elaboración propia.

El elemento más llamativo de los resultados es el hecho de que a partir de mayo de 2009, los empresarios parecen incorporar información macroeconómica relevante, la cual no es incorporada por los demás estratos. Haciendo la salvedad de que el nivel de las expectativas de inflación sí ayuda a predecir los errores (tal y como se presentó previamente), la evidencia indica que solamente este estrato sobrevive a esta prueba muy específica de racionalidad. Este resultado es positivo dado que en la nueva muestra que comenzó a utilizar recientemente los empresarios constituyen el grupo más numeroso del conjunto de agentes consultados ${ }^{21}$.

$21 \quad$ Si se utiliza el quiebre estructural alternativo (12 meses después), los resultados son similares a excepción de que algunas variables dejan de ser significativas. No obstante, persiste el resultado cualitativo de que solamente el estrato de empresarios parece incorporar información macroeconómica disponible. 


\section{Dispersión de las expectativas}

Una de las observaciones que se han realizado en la literatura es la reducción en la dispersión de las expectativas conforme el nivel de inflación es menor y una convergencia hacia la media de la encuesta conforme pasa el tiempo. La medida de dispersión utilizada como parámetro en el presente documento es el rango intercuartil ${ }^{22}$ de las expectativas de inflación. En el gráfico 5 se presenta esta medición para cada uno de los estratos de la encuesta, $y$ se muestra como referencia, el nivel de inflación para el mes en el que se manifiestan las expectativas.

GRÁFICO 5

TASA DE INFLACIÓN Y RANGO INTERCUARTIL DE CADA ESTRATO

ENERO 2009 - ABRIL 2013
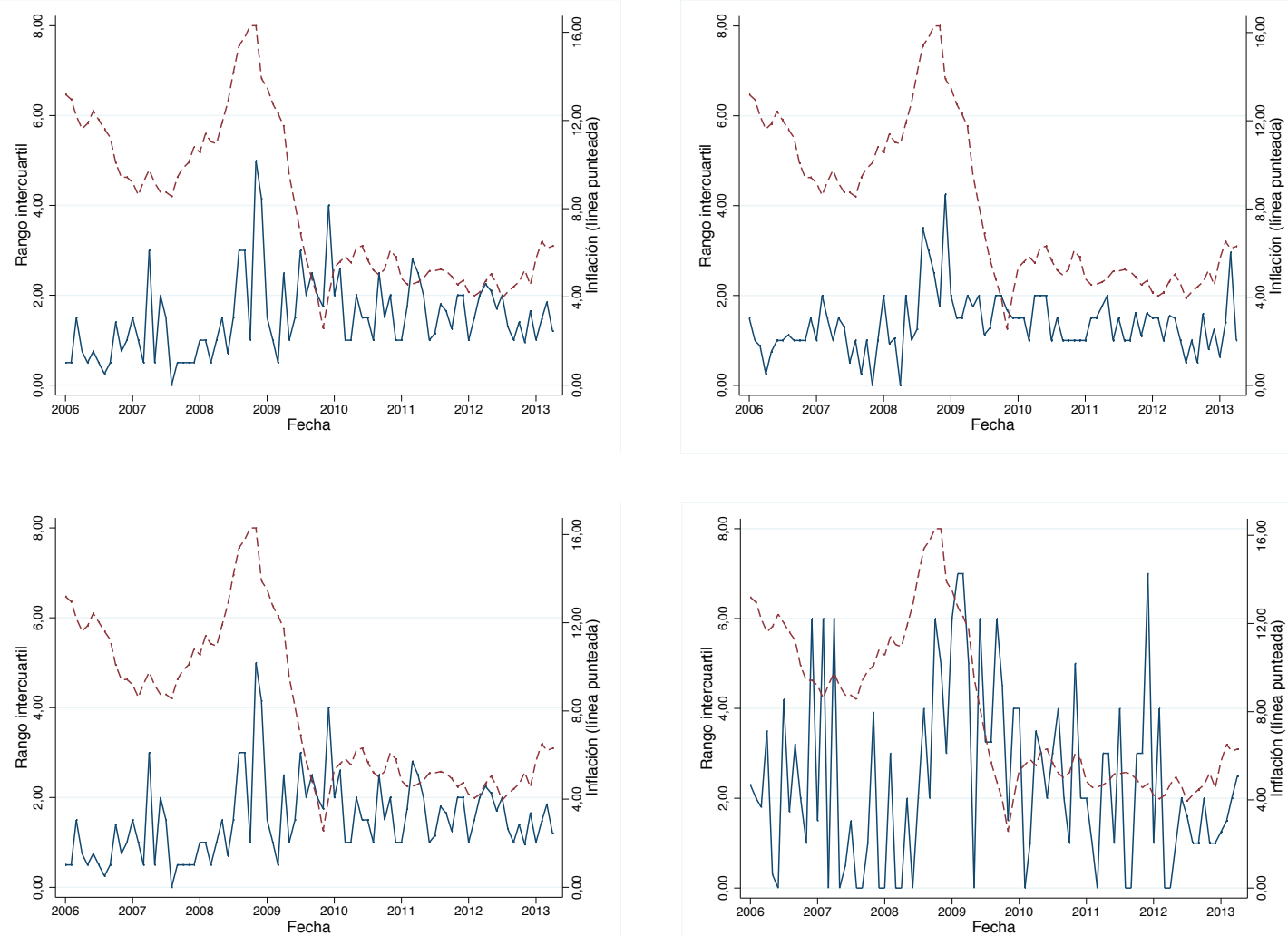

Fuente: Elaboración propia.

Mediante este gráfico se puede observar que tras el cambio estructural se da una lenta reducción de la dispersión en la mayoría de estratos, siendo la única excepción el de empresarios, cuya mayor volatilidad en comparación a los otros grupos, se debe al tamaño reducido de la muestra. Para el periodo previo al quiebre estructural, existe una clara correlación entre el nivel de inflación y el grado de dispersión para todos los estratos. En este sentido, se reportan coeficientes positivos, tanto de correlación como de regresión lineal, entre las dos variables. 
Esta relación se mantiene en casi todos los estratos una vez que se amplía el periodo a toda la muestra (ver gráfico 10 del Anexo C), con excepción del estrato de consultores, que presenta una correlación negativa.

Adicional a esta relación directa entre inflación y el desacuerdo en las expectativas de los agentes con respecto a la variación de los precios, en el gráfico 6 se muestra que cuanto mayor sea la variación de la inflación interanual, tanto al alza (positiva) como a la baja (negativa), mayor será la dispersión de las expectativas. Cabe recalcar que esta correlación es mayor para los casos en los que la inflación varía de manera positiva, que en los que varía negativamente, es decir, que el rango intercuartil aumenta en mayor medida, cuando la inflación interanual está a la alza, en comparación a cuando está a la baja. Gráficamente se puede realizar la misma inferencia, debido a que en la mayoría de los estratos la línea vinculada con las variaciones interanuales de inflación es más inclinada para los casos positivos que para los casos negativos. De esta forma, en el resultado de las regresiones y de los análisis de correlación se observa que todos los estratos, excepto el estrato académico, que no muestra un comportamiento claro, la dispersión de las expectativas aumenta ante variaciones positivas de la inflación.

\section{GRÁFICO 6 \\ RELACIÓN DEL RANGO INTERCUARTIL DE LA EXPECTATIVA DE CADA ESTRATO Y DIFERENCIA INTERANUAL DE LA INFLACIÓN OBSERVADA \\ ENERO 2006 - ABRIL 2013}
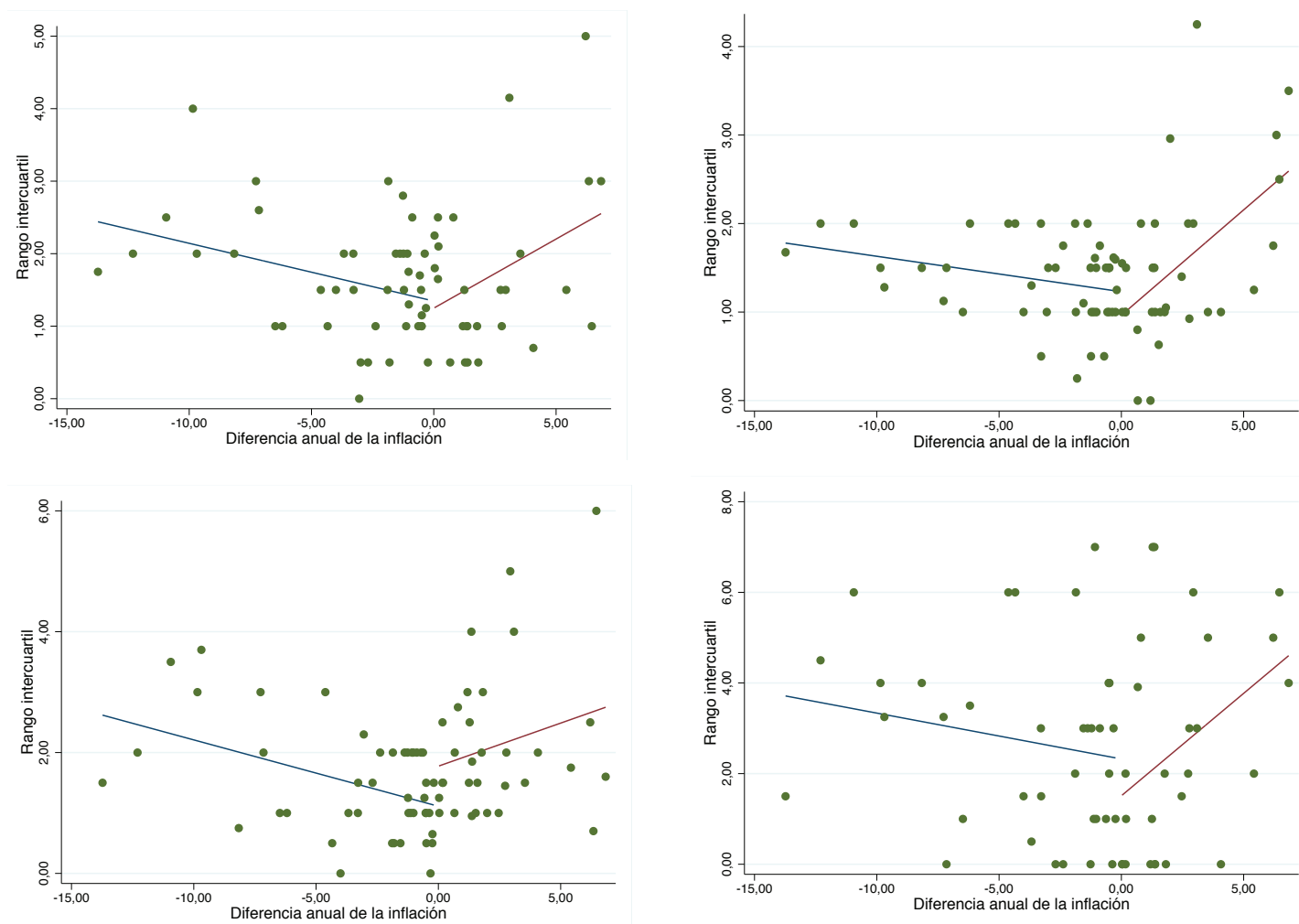

Fuente: Elaboración propia. 
Otro análisis que se puede realizar con relación a los determinantes de la dispersión en las expectativas de inflación entre los agentes, es el vinculado al rango intercuartil de la inflación según componentes $^{23}$. En este sentido, existe una correlación positiva entre el rango intercuartil de ambas variables. En el gráfico 11 del Anexo C, se observa esta relación gráficamente. Al analizar esta información mediante una regresión y un cálculo de correlación, se infiere que esta relación no es significativa estadísticamente en los estratos de consultores y empresarios. En los estratos que restan, en el de analistas y académicos, sí se presenta una relación de correlación positiva entre ambas variables, lo cual permite inferir que una mayor dispersión entre la evolución de los componentes del IPC contribuye a acentuar las diferencias entre las expectativas intraestrato.

\section{Hipótesis sobre formación de expectativas}

Resulta evidente, en los análisis presentados, que existen sesgos significativos y persistentes a lo largo del tiempo en la formación de expectativas de prácticamente todos los agentes. Una posible explicación es que la formación de expectativas de variación de precios de los distintos estratos esté correlacionada entre sí, siendo que las expectativas de algún grupo podrían influir sobre la formación de las expectativas de los otros ${ }^{24}$.

En el caso de la encuesta de expectativas de inflación, el estrato de consultores es el que representaría de una manera más apropiada a este tipo de individuos con capacidad de formar expectativas. Sin embargo, no es posible encontrar una causalidad (en el sentido de Granger) de la serie de los consultores hacia la expectativa promedio del resto de los encuestados ${ }^{25}$, cuando se considera la totalidad de la muestra. Sin embargo, cuando solamente se considera la primera parte del periodo (previo al quiebre estructural), es posible encontrar causalidad de la serie de consultores hacia la serie de los demás estratos incluidos en la encuesta. Es posible apreciar gráficamente que la serie en efecto antecede en algunos momentos la de los demás agentes consultados (ver gráfico 3).

No obstante, cuando se considera solamente el último periodo, no es posible encontrar causalidad en un sentido solamente. Tal y como se menciona anteriormente, el resultado es el mismo cuando se consideran la totalidad de la muestra. Esto permite inferir que previo a mayo de 2009, el estrato de consultores tenía cierta influencia sobre lo que manifestaban los demás agentes $^{26}$. Si se considera que la serie de consultores refleja la información que es transmitida a los demás agentes de la economía, se podría afirmar que este proceso de formación de expectativas pudo haber sido válido previo a la desinflación de 2009, pero ha dejado de serlo en años recientes. Actualmente, en la economía costarricense, esta influencia ha dejado de ser estadísticamente significativa, ya que el proceso de formación de expectativas de los agentes parece ser un proceso simultáneo.

$23 \quad$ Este cálculo se realiza a partir de la base de datos que contiene la variación de precios de todos artículos de la canasta que utiliza el INEC para calcular mensualmente el IPC. A partir de la variación mensual de la variación ponderada de cada uno de estos productos se calcula el rango intercuartil.

Una hipótesis plausible sería que el criterio de estratos o grupos formadores de opinión en temas económicos influya mediante seminarios, publicaciones, o a partir de la exposición de sus opiniones en diversos medios comunicación, en las expectativas del resto de la población.

Este análisis de causalidad se realiza utilizando en cada ejercicio la serie de consultores y la de cada uno de los demás estratos. seminarios a los cuales asisten, por ejemplo, los empresarios y los analistas bursátiles. 


\section{Hipótesis sobre expectativas adaptativas}

Los resultados presentados previamente permiten rechazar la hipótesis de expectativas racionales expresada en diversas formas. Adicionalmente, tomando en cuenta la totalidad de la muestra, los pronósticos realizados por el estrato de consultores coinciden con las expectativas de los demás agentes, pero no la anteceden, por lo cual también es posible descartar una influencia de un grupo particular sobre el resto de personas consultadas en la muestra para el periodo más reciente. Un supuesto más sencillo sería suponer expectativas adaptativas. Mankiw et al. (2004) realizan esta prueba para los datos de las encuestas de expectativas estadounidenses y rechazan la hipótesis de formación de las mismas de manera adaptativa al encontrar que alguna información macroeconómica resulta relevante estadísticamente para su establecimiento.

Una manera muy sencilla de someter a prueba esta hipótesis es utilizar la Ecuación (11), en la cual se incluyen datos de producción medida por medio de la variación interanual del IMAE $(Y)$ y tasa de interés $(i)$ medida en el mes durante el cual se realizan las consultas. Adicionalmente se introducen ambas variables con un trimestre de rezago. También se incluyen medidas de inflación de los Estados Unidos (), y una medida de un índice de precios del petróleo (), que promedia precios de diferentes tipos de combustible. Además, se incluyen $L=8$ rezagos de la inflación trimestral anualizada ${ }^{27}$. Por lo tanto, se consideran dos años completos de información relativa a la inflación. Se incluye también el dato de la meta de inflación anunciada por el BCCR $\left(\pi_{t}^{m}\right)^{28}$.

Finalmente, se incluyen cuatro variables dicotómicas (denotadas con $d$ ), las cuales toman un valor de uno para diferentes periodos en los cuales hubo cambios de la meta anunciada por el BCCR. Representan, en el orden respectivo, un cambio de la meta hacia un valor superior, un abandono de la meta hacia un valor superior, un cambio de la meta hacia un nivel inferior $y$ un abandono de la meta hacia un valor inferior. Estos valores consideran la información que tenían los agentes en el momento de formular la expectativa para un año después ${ }^{29}$.

$$
\begin{gathered}
E_{t+12} \pi_{t}=\alpha+\beta(L) \pi_{t}^{t r i m}+\gamma Y_{t}+\delta Y_{t-3}+\eta i_{t}+\varphi i_{t-3}+\psi \pi_{t}^{U S A}+\xi P_{t}^{P e t}+\mu \pi_{t}^{m}+ \\
\kappa_{1} d_{1}+\kappa_{2} d_{2}+\kappa_{3} d_{3}+\kappa_{4} d_{4}+\varepsilon_{t}
\end{gathered}
$$

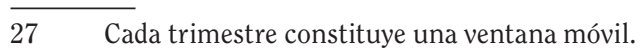

28 Esta variable se construyó considerando la información respecto a la inflación meta un año adelante proveniente de los anuncios del BCCR que los agentes tenían en el momento preciso de realizar el pronóstico. esta información a partir de la encuesta de febrero, por lo que en enero aún no conocen el cambio de meta. 
CUADRO 9

RESULTADO DE MCO DE ECUACIÓN (11)

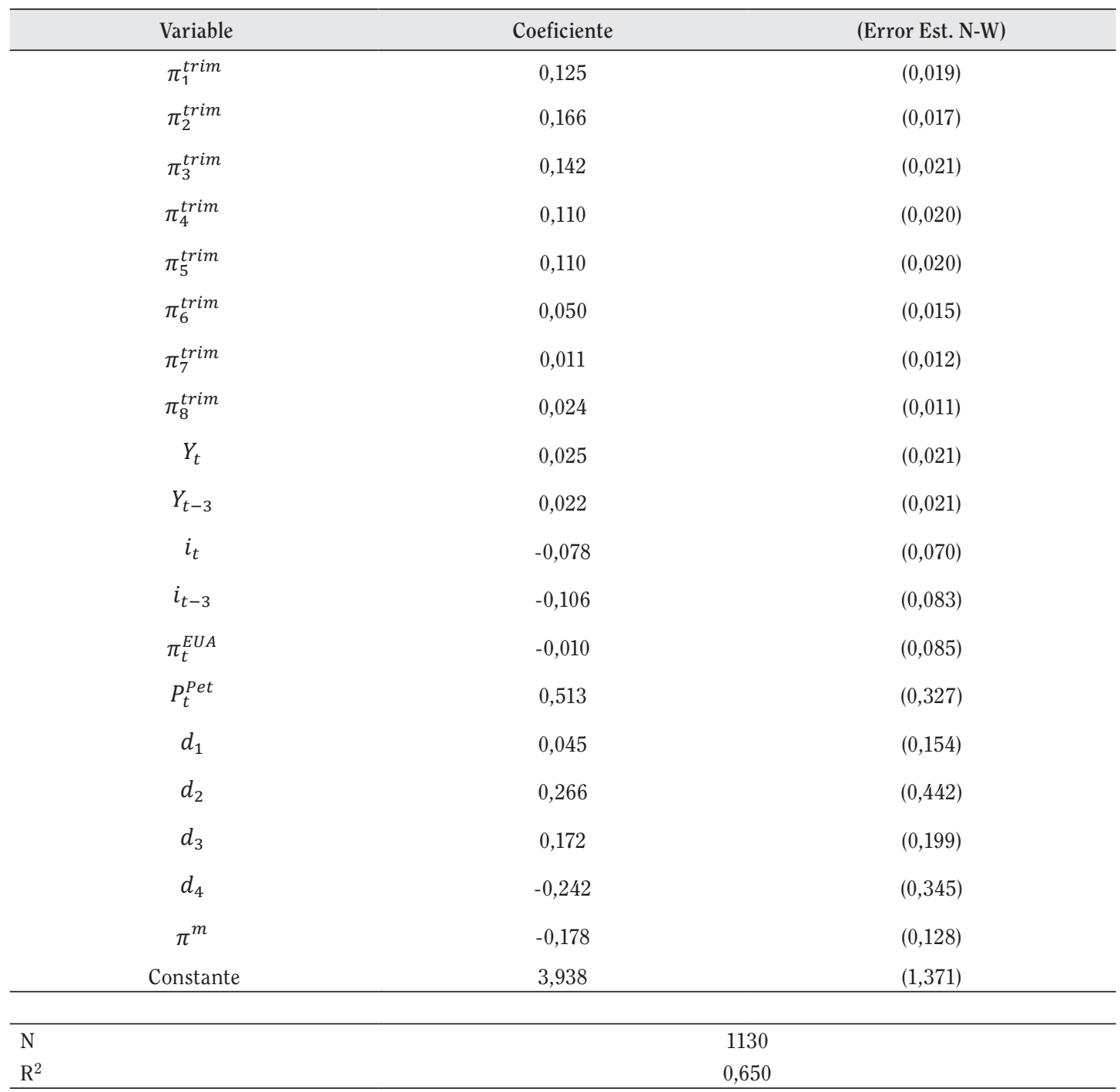

Nivel de significancia : †: $10 \% *: 5 \% * *: 1 \%$

Fuente: Elaboración propia.

Los resultados se presentan en el cuadro 9, en el cual se puede observar que solamente algunos de los valores de inflación pasada resultan relevantes para la formación de las expectativas de inflación. Siete trimestres de rezago son incorporados por los agentes, mientras que la meta anunciada por el BCCR no es estadísticamente significativa.

El elemento crucial de esta estimación es que al realizar la prueba $F$ correspondiente, a un nivel de significancia de menos de un $1 \%$ se puede rechazar la hipótesis nula de $\gamma=\delta=\eta=\varphi=\psi=\xi=\mu=\kappa_{1}=\kappa_{2}=\kappa_{3}=\kappa_{4}=0$. Esto significa que, a pesar de haber descartado la idea de que los agentes forman expectativas racionales, tampoco es posible interpretar que las expectativas de los agentes se forman de una manera ingenua como en un 
modelo de expectativas adaptativas. Este resultado es el mismo que obtienen Mankiw et al. (2004) para la economía estadounidense, aunque en el caso de ellos la información que se incorpora es relativa a tasas de interés y desempleo ${ }^{30}$. En el caso de Costa Rica, los agentes parecen incorporar alguna información de variables macroeconómicas en la formulación de las expectativas de inflación. Por ejemplo, los precios de petróleo son cercanos a ser significativos al 10\%, y estos posiblemente impactan en el mediano plazo el resto de precios de la economía, por lo que los agentes podrían tomarlos en consideración.

Es destacable, sin embargo, que los agentes no parecen incorporar cambios en los anuncios realizados con respecto a la meta, ni tampoco la meta en sí, como elementos relevantes para la formación de las expectativas que manifiestan. Como una prueba de cuán robusto es el resultado de que el coeficiente de la variable meta de inflación no sea significativo, se realizaron 41 regresiones diferentes en las que el periodo en consideración consistió de ventanas de 4 años de extensión. Los resultados son exactamente los mismos, en ningún caso hay muestras de algún grado de significancia de estos coeficientes. En el Anexo D se puede observar el gráfico respectivo.

\section{MODELO BASE PARA PROYECTAR LAS EXPECTATIVAS DE INFLACIÓN}

Cuando se consideran solamente los trimestres que resultan significativos, los resultados indican que la inflación de los trimestres previos que resultan significativos es incorporada en un 64,9\% en las expectativas. En el caso de EUA se indica un valor de 70\%, lo cuál refleja una alta incorporación de la inflación reciente en la formación de expectativas. Si se incluyen en una regresión los trimestres que resultan significativos, además del índice de precios del petróleo, el modelo resultante tiene las variables presentadas en el cuadro 10.

CUADRO 10

RESULTADO DE MCO DE ECUACIÓN (11), CONJUNTO REDUCIDO DE VARIABLES

\begin{tabular}{lcc}
\hline Variable & Coeficiente & (Error Est. N-W) \\
\hline$\pi_{1}^{\text {trim }}$ & 0,151 & $(0,014)$ \\
$\pi_{2}^{\text {trim }}$ & 0,160 & $(0,016)$ \\
$\pi_{3}^{\text {trim }}$ & 0,124 & $(0,013)$ \\
$\pi_{4}^{\text {trim }}$ & 0,076 & $(0,011)$ \\
$\pi_{5}^{\text {trim }}$ & 0,070 & $(0,013)$ \\
$\pi_{6}^{\text {trim }}$ & 0,028 & $(0,010)$ \\
$\pi_{8}^{\text {trim }}$ & 0,039 & $(0,012)$ \\
$P_{t}^{\text {Pet }}$ & 0,765 & $(0,233)$ \\
Constante & 1,750 & $(0,519)$ \\
\hline
\end{tabular}

\begin{tabular}{ll}
\hline $\mathrm{N}$ & 1190 \\
$\mathrm{R}^{2}$ & 0,635 \\
\hline
\end{tabular}

Nivel de significancia : †: $10 \% *: 5 \% * *: 1 \%$

Fuente: Elaboración propia.

30 El dato de desempleo no se incorpora ya que la metodología para extender esta variable al periodo completo en frecuencia mensual es menos confiable ya que antes de 2010 utiliza datos anuales. 
Este modelo simple puede utilizarse como base para generar proyecciones para la expectativa de inflación, lo cuál podría ser un insumo importante para los modelos que utiliza el BCCR en el análisis de la política monetaria. No se podría descartar que sea necesario incluir alguna otra variable.

En el caso de querer realizar un pronóstico de corto plazo de la serie de expectativas de inflación, se sugiere incluir, además de las variables propuestas, un componente autorregresivo de orden 1. Las proyecciones que se pueden realizar con esta información son bastante acertadas, tal y como se puede apreciar en el gráfico 7.

\section{GRÁFICO 7}

\section{MODELO PARA PROYECTAR EXPECTATIVAS EN CORTO PLAZO, INCLUYE COMPONENTE AR(1)}

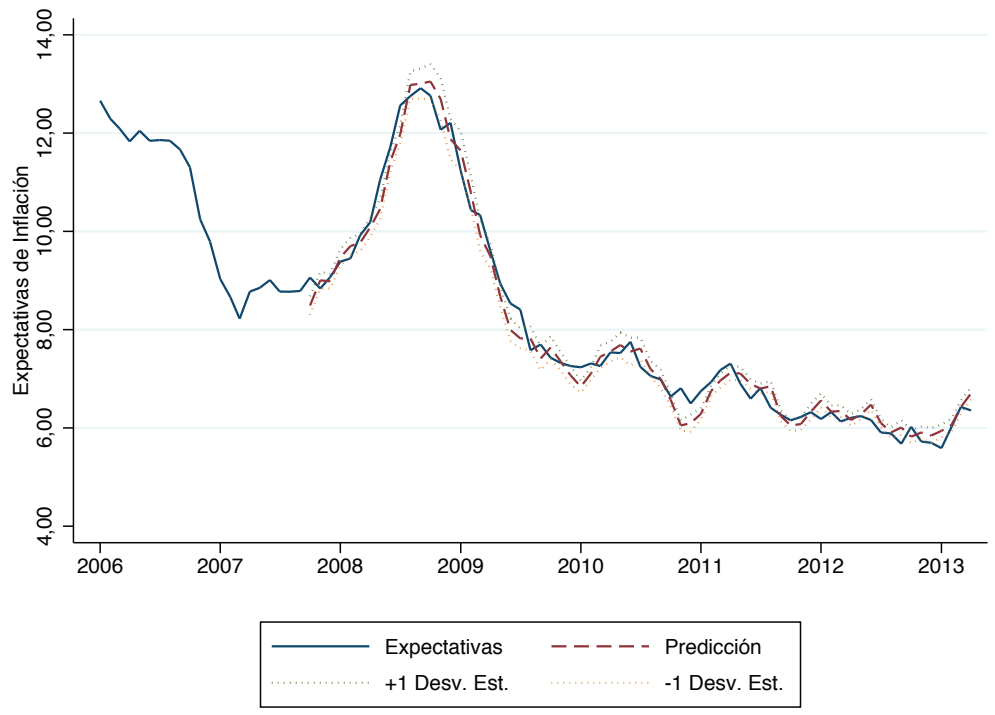

Fuente: Elaboración propia.

Por otra parte, si se desea realizar un pronóstico de mediano plazo de dónde se ubicarían las expectativas de inflación, se sugiere incluir solamente las variables detalladas en el cuadro 10. Si se supone que a partir de este instante la inflación trimestral anualizada es $3 \%$ y que el índice de precios de petróleo mantienen los valores promedio observados en la muestra se puede realizar un ejercicio de proyección. Al utilizar un horizonte de 24 meses (cuyos resultados se pueden apreciar en el Anexo E), la expectativa de la población logra reducirse solamente hasta 5,22 al concluir el horizonte de proyección ${ }^{31}$.

Esta proyección está, sin duda, sujeta a la crítica de Lucas. Si el BCCR modifica sus políticas para lograr en el corto plazo una inflación de 3\%, los parámetros no necesariamente se mantendrían invariables cuando los agentes incorporen esta nueva información. Sin embargo, resulta un

31 Es posible realizar el mismo tipo de proyección suponiendo que la información está disponible hasta cierto momento en el tiempo y evaluar la capacidad de proyección fuera de muestra. Si se realiza la misma regresión, pero solamente utilizando los datos hasta diciembre de 2011 y se incorpora en la proyección los datos que los agentes podrían observar en el momento de manifestar la expectativa, las proyecciones hubiesen sido ligeramente superiores, aunque menos precisas, especialmente para el periodo ya transcurrido (enero 2011 a abril 2013). Para los dos años siguientes de proyección los parámetros resultantes indican que la expectativa de inflación se ubicaría en $5,26 \%$ a mediados de 2015. El detalle de estas proyecciones se puede observar en el gráfico 15 del Anexo E. 
ejercicio interesante para comprender las limitaciones actuales con las que cuenta el BCCR para reducir la expectativa promedio que manifiestan las personas a quienes se consulta.

Como ejercicio adicional, a la luz del hecho de que la inflación trimestral anualizada se ha mantenido en nivel alrededor de 4,90\% a partir de enero de 2011, se puede mantener este nivel para los siguientes dos años. A partir de esta dinámica, la proyección indica que la expectativa de inflación más bien subiría para estabilizarse en un nivel de 6,45\%. De esta forma, se puede concluir que para que el BCCR logre reducir la expectativa de inflación a niveles dentro del rango de meta, es necesario que la inflación sea sumamente baja por mucho tiempo o, alternativamente, que los agentes incorporen el anuncio de la meta como la fuente más importante para la formación de sus expectativas. Si este cambio en el comportamiento de los agentes resulta en mejores predicciones, la situación estaría cambiando hacia una en la que el modelo de expectativas racionales es el más apropiado.

\section{CONCLUSIONES Y RECOMENDACIONES}

La encuesta de expectativas de inflación para la economía costarricense muestra evidencia clara de sesgos en los pronósticos. Estos sesgos son persistentes y hay evidencia leve de que los agentes incorporan la información disponible con respecto de sus errores previos para formular nuevos pronósticos ${ }^{32}$. Estas características parecen ser más importantes en el periodo posterior al quiebre estructural en los datos de inflación para el caso costarricense, periodo que se extiende hasta el final de la muestra.

Por lo tanto, es posible descartar la hipótesis de expectativas racionales en los agentes consultados. Esquivel (2009) encuentra un resultado similar para el periodo comprendido entre el cuarto trimestre de 1999 y el cuarto trimestre de 2008 en Costa Rica. Sin embargo, la persistencia de errores tampoco parece estar relacionada solamente con inflación pasada, como un modelo adaptativo sugeriría, pues los agentes incorporan alguna información macroeconómica disponible para formular sus expectativas.

Resulta evidente que todos los agentes, sin importar a qué estrato de la muestra pertenezcan, forman sus expectativas de una manera muy parecida debido a que utilizan la misma información y los sesgos son sumamente similares. Es descartable que algún estrato forme sus expectativas de una manera más acertada que otros. Adicionalmente, no pareciera ocurrir que los agentes manifiesten pronósticos en los que evidencien, para el periodo a partir de mayo 2009, que se ven influidos por la opinión de los consultores. Tomando en cuenta el último subperiodo así como la totalidad de la muestra, es usual que los pronósticos propios de los consultores estén en consonancia con el resto de los agentes consultados.

No obstante, es interesante notar que los resultados sugieren (aun cuando no hay suficiente información estadística para tener total seguridad al respecto) que el estrato de empresarios ha tenido sesgos más grandes, pero son los individuos que menor persistencia presentan en los errores. Adicionalmente, los empresarios son los únicos para quienes se puede descartar que la incorporación de algunos datos macroeconómicos pudieran ayudar a reducir el error. Esto es un elemento positivo dado que la nueva muestra, que se implementó a partir de junio de 2012, tiene una mayor proporción de agentes pertenecientes a este estrato.

Es relevante sugerir el desarrollo de una investigación que trasciende los objetivos del presente documento. Resulta crucial comprender cuál es el proceso de formación de expectativas con un modelo alternativo a los que se presentan en este documento. Una posibilidad es la modelación

32 El no rechazo de esta hipótesis de racionalidad ocurre solamente para algunos estratos cuando se considera el periodo alternativo posterior a mayo de 2010 . 
de formación de expectativas con rigidez de información (sticky-information model), tal y como desarrollan Mankiw et al. (2004). En este tipo de modelo los agentes deben reoptimizar las predicciones que realizan, pero solamente una pequeña fracción de agentes realiza esta actualización en un momento determinado. Este tipo de modelo genera dispersión en los pronósticos de diferentes agentes y ocasiona un proceso lento de adopción de la información que se utiliza para optimizar el pronóstico. Estas predicciones serían consecuentes con una alta dispersión en las expectativas que se reduce paulatinamente $y$ con un proceso lento para reducir los errores del pasado, características que están presentes en la información de la encuesta que realiza el BCCR.

Una alternativa adicional es la utilización de modelos de racionalidad acotada, tales como los desarrollados por Evans y Honkapohja ${ }^{33}$, entre otros. En este tipo de modelos los agentes se comportan como si tuvieran expectativas racionales mientras se cumplan determinadas condiciones, pero es posible que seleccionen una alternativa "no-racional" para la formación de expectativas en el largo plazo, lo cual depende de parámetros del modelo.

\section{REFERENCIAS}

Blinder, A. S. (1999). Central bank credibility: Why do we care? How do we build it? NBER Working Papers 7161, National Bureau of Economic Research, Inc.

Borraz, F. \& Gianelli, D. (2011). Un análisis de comportamiento a nivel de agente de la encuesta de expectativas de inflación del BCU. Documentos de Trabajo (working papers) 0111, Departmento de Economía, FCS - UDELAR.

Cerisola, M. \& Gelos, R. G. (2005). What drives inflation expectations in Brazil? An empirical analysis. IMF Working Papers 05/109, International Monetary Fund.

Departamento de Investigación Económica (2012). Plan de investigación 2012-2013. Documento DEC-DIE-02-2012, División Económica, BCCR, San José, Costa Rica.

Diebold, F. X. \& Lopez, J. A. (1995). Forecast evaluation and combination. Technical report.

Esquivel, M. (2009). Hipótesis de racionalidad y modelación del proceso de formación de expectativas de inflación en Costa Rica. Documento de Investigación DEC-DIE-024-2009, Departamento de Investigación Económica, BCCR.

Evans, G. W., Honkapohja, S., Sargent, T., \& Williams, N. (2012). Bayesian model averaging, learning and model selection. CDMAWorking Paper Series 1203, Centre for Dynamic Macroeconomic Analysis.

Johnson, D. (1998). The credibility of monetary policy: International evidence based on surveys of expected inflation. Research document, Bank of Canada, Canada.

Keane, M. P. \& Runkle, D. E. (1990). Testing the rationality of price forecasts: New evidence from panel data. American Economic Review, 80(4), 714-35.

Kydland, F. E. \& Prescott, E. C. (1977). Rules rather than discretion: The inconsistency of optimal plans. Journal of Political Economy, 85(3), 473-91.

Leyva Jiménez, G. A. (2012). Errores sistemáticos en los pronósticos del panel de analistas privados en Guatemala: ¿̇son irracionales sus pronósticos de inflación? Documento de Trabajo 125, Banco de Guatemala.

Lucas, R. J. (1976). Econometric policy evaluation: A critique. Carnegie-Rochester Conference Series on Public Policy, 1(1), 19-46.

Mankiw, N. G., Reis, R., \& Wolfers, J. (2004). Disagreement about inflation expectations. In NBER Macroeconomics Annual 2003, Volume 18, NBER Chapters (pp. 209-270). National Bureau of Economic Research, Inc.

33 Para un ejemplo reciente, ver Evans, Honkapohja, Sargent y Williams (2012). 
Muñoz, E. \& Torres, C. (2006). Un modelo de formación de expectativas de inflación para Costa Rica. Documento de Investigación DEC-DIE-003-2006, Departamento de Investigación Económica, BCCR.

Rebucci, A. \& Rossi, M. (2006). Measuring disinflation credibility in emerging markets: A bayesian approach with an application to Turkey's IMF-supported program. Economics Bulletin, 6(11), $1-8$.

Torres, C. (2012). Costa Rica: Determinación de cambios estructurales en el nivel de la tasa de inflación: periodo 1997-2011. Documento de Trabajo 02-2012, Departamento de Investigación Económica, BCCR.

Urich, T. \& Wachtel, P. (1984). The structure of expectations of the weekly money supply announcement. Journal of Monetary Economics, 13, 183-94.

Wooldridge, J. M. (2002). Econometric Analysis of Cross Section and Panel Data. The MIT Press. 


\section{ANEXOS}

\section{A: DATOS DE LA ENCUESTA DE EXPECTATIVAS DE INFLACIÓN}

GRAFICO 8

EXPECTATIVAS DE INFLACIÓN DESAGREGADAS POR ESTRATO

DATOS RECOPILADOS DE ENERO 2010 A ABRIL 2013

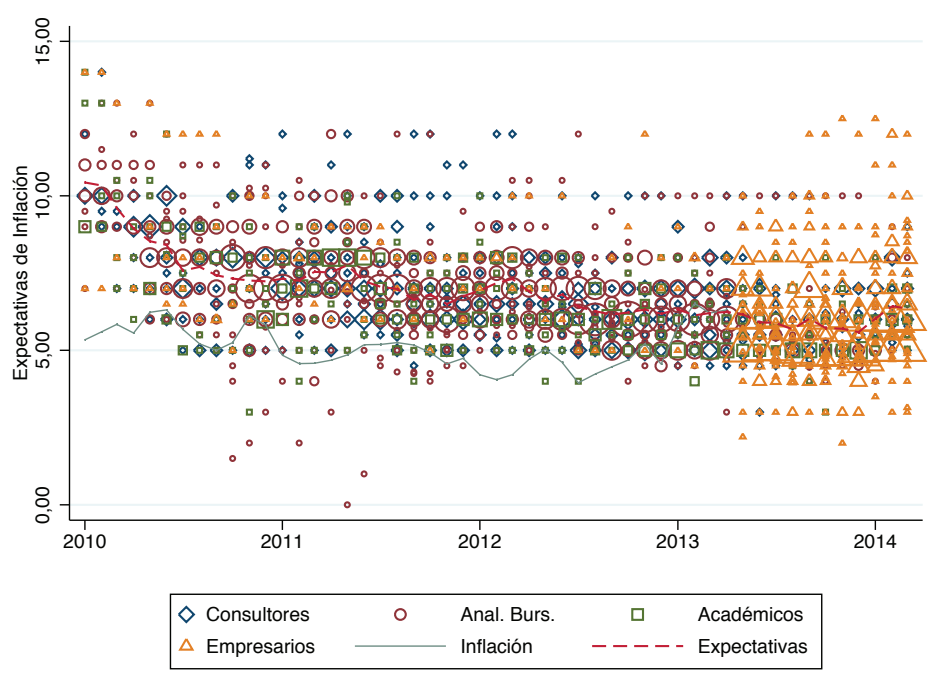

Fuente: Elaboración propia.

B: ERRORES DE PREDICCIÓN

GRAFICO 9

EXPECTATIVA DE INFLACI'ON Y ERROR

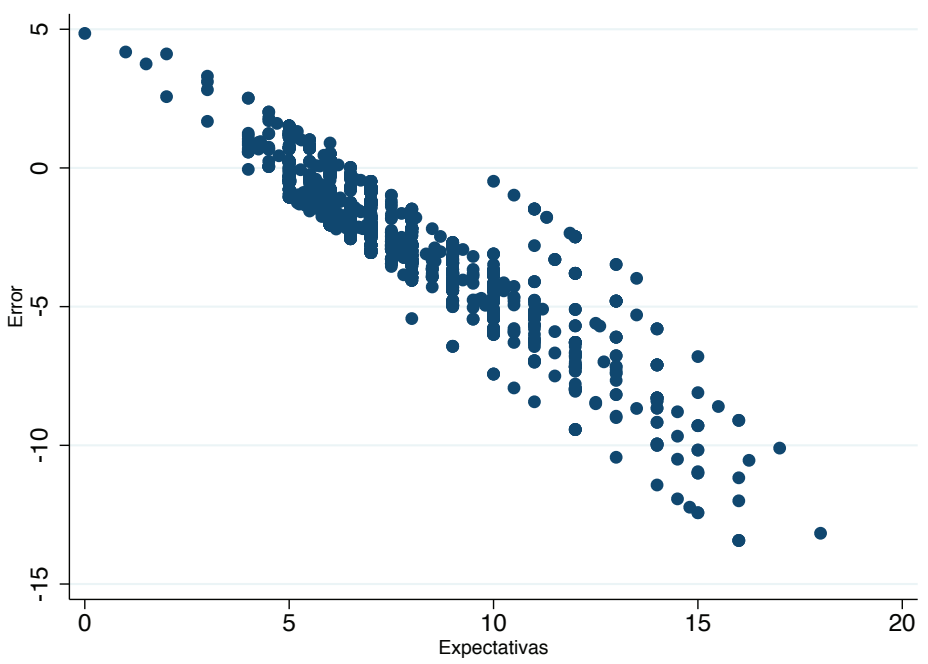

Fuente: Elaboración propia. 


\section{C: DISPERSIÓN E INFLACIÓN}

\section{GRAFICO 10}

RELACIÓN ENTRE RANGO INTERCUARTIL DE CADA ESTRATO E INFLACIÓN ENERO 2006 - ABRIL 2013
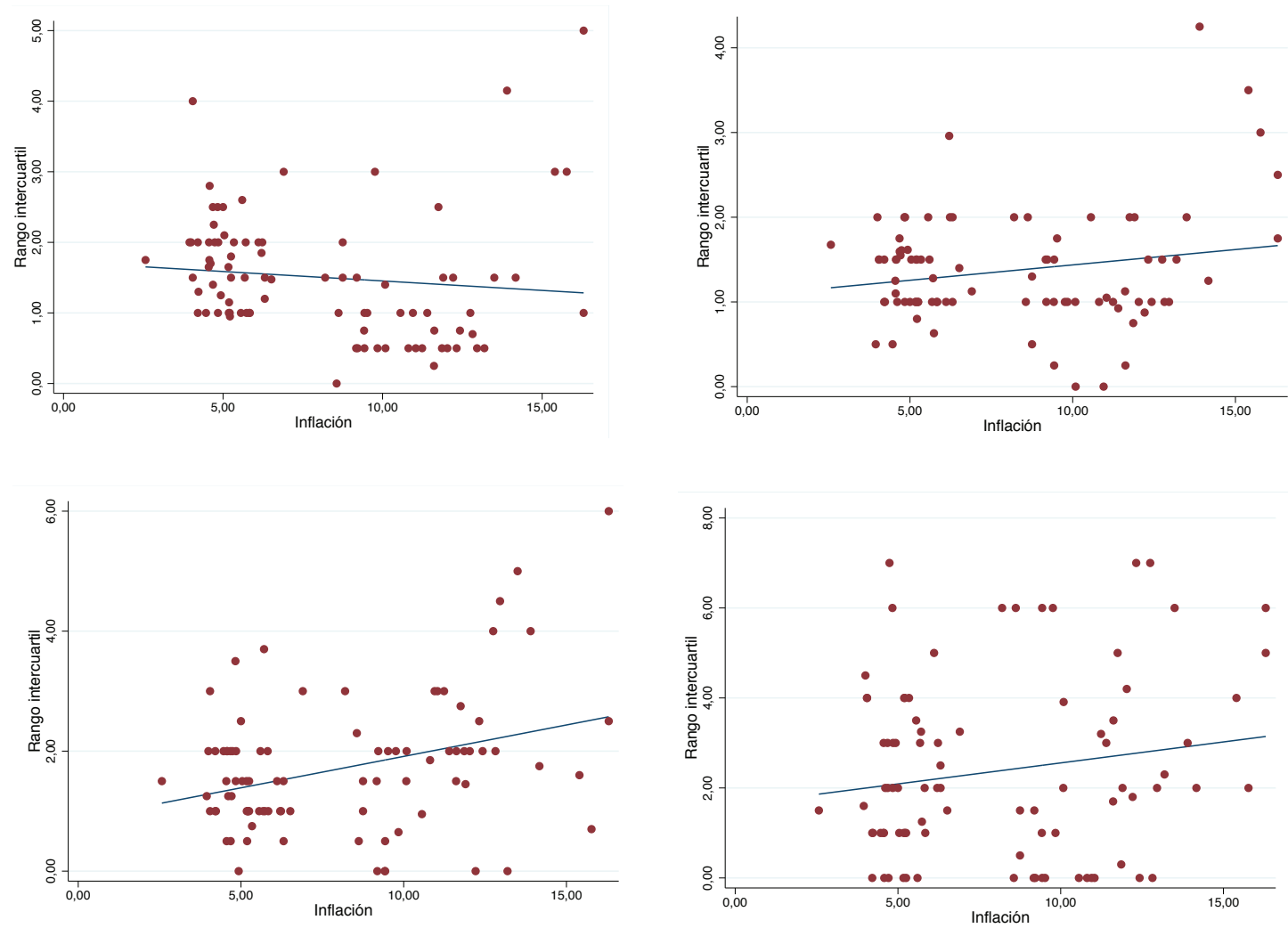

Fuente: Elaboración propia. 

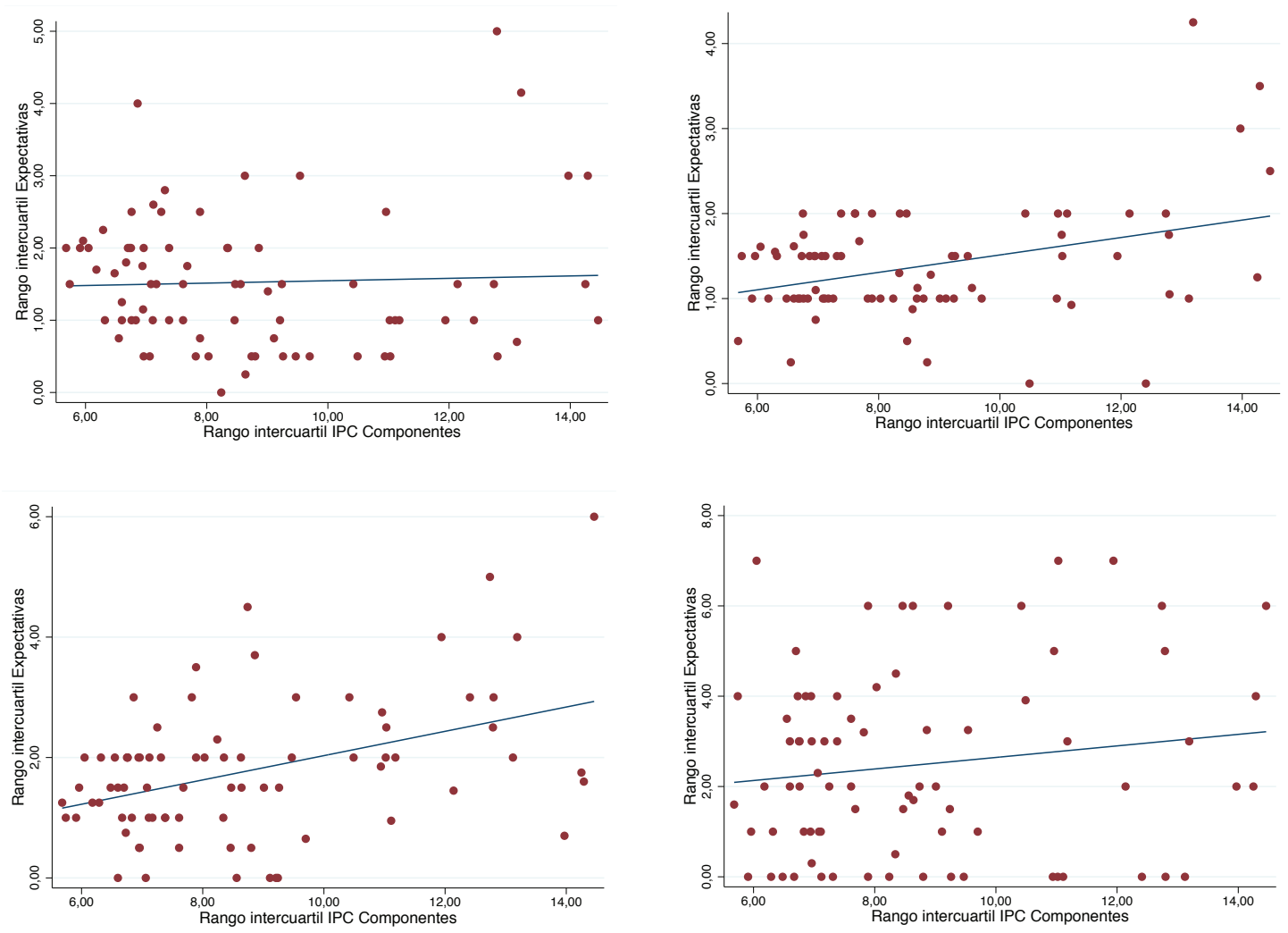

Fuente: Elaboración propia. 
D: ESTABILIDAD DE COEFICIENTE DE META DE INFLACIÓN.

GRÁFICO 12

ESTABILIDAD DE COEFICIENTE .

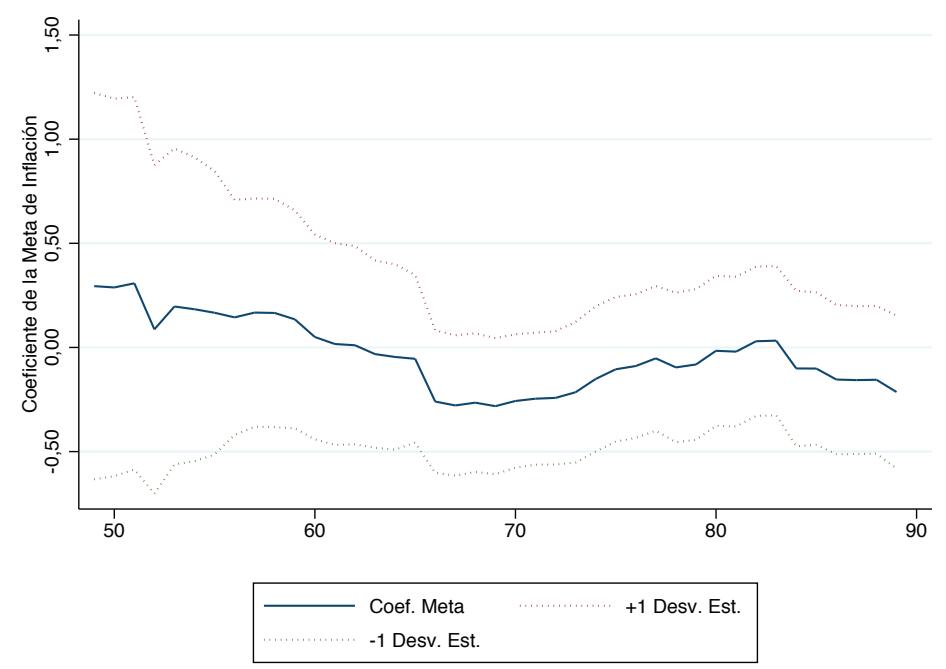

Fuente: Elaboración propia.

\section{E: PREDICCIÓN DE EXPECTATIVAS DE INFLACIÓN}

GRÁFICO 13

PROYECCIÓN CON BASE EN MODELO BASE DE EXPECTATIVAS DE INFLACIÓN, INFLACIÓN =3 \%.

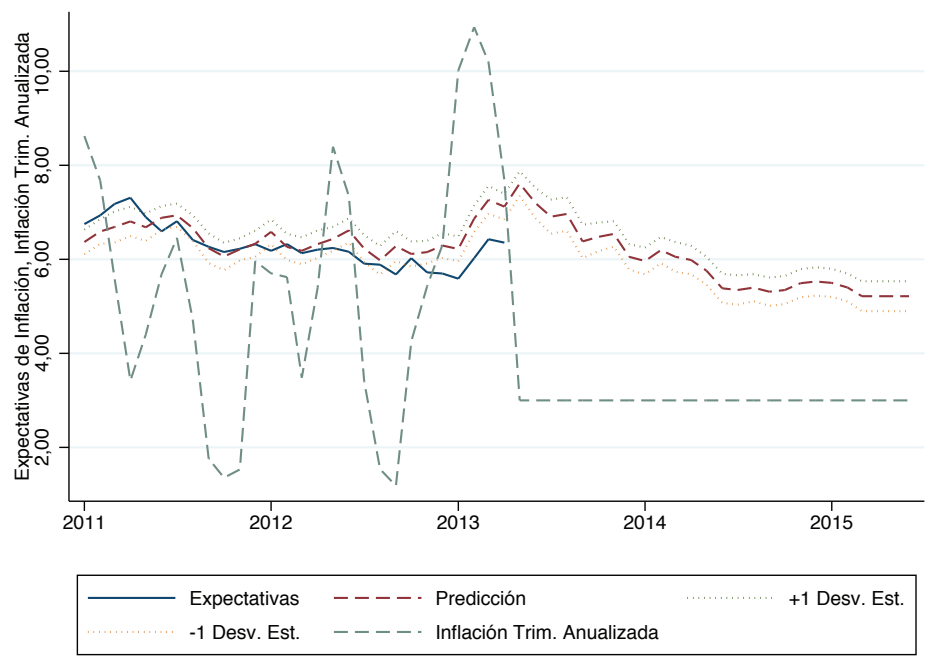

Fuente: Elaboración propia. 


\section{GRÁFICO 14}

PROYECCIÓN CON BASE EN MODELO BASE DE EXPECTATIVAS DE INFLACIÓN, INFLACIÓN = 4,90.

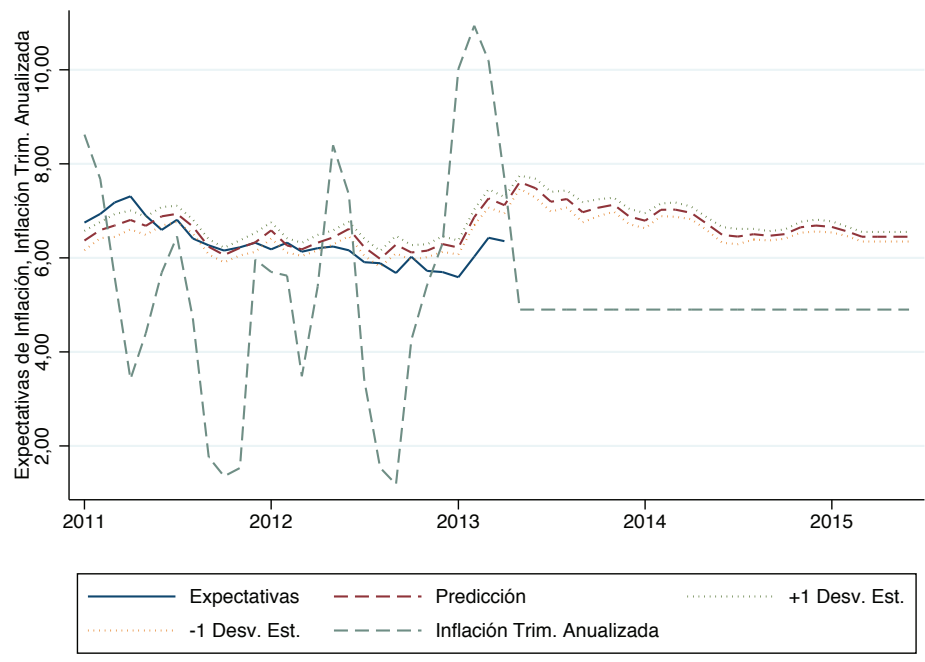

Fuente: Elaboración propia.

\section{GRÁFICO 15}

PREDICCIÓN FUERA DE MUESTRA DE MODELO BASE DE EXPECTATIVAS DE INFLACIÓN. MUESTRA CON DATOS HASTA DICIEMBRE 2011.

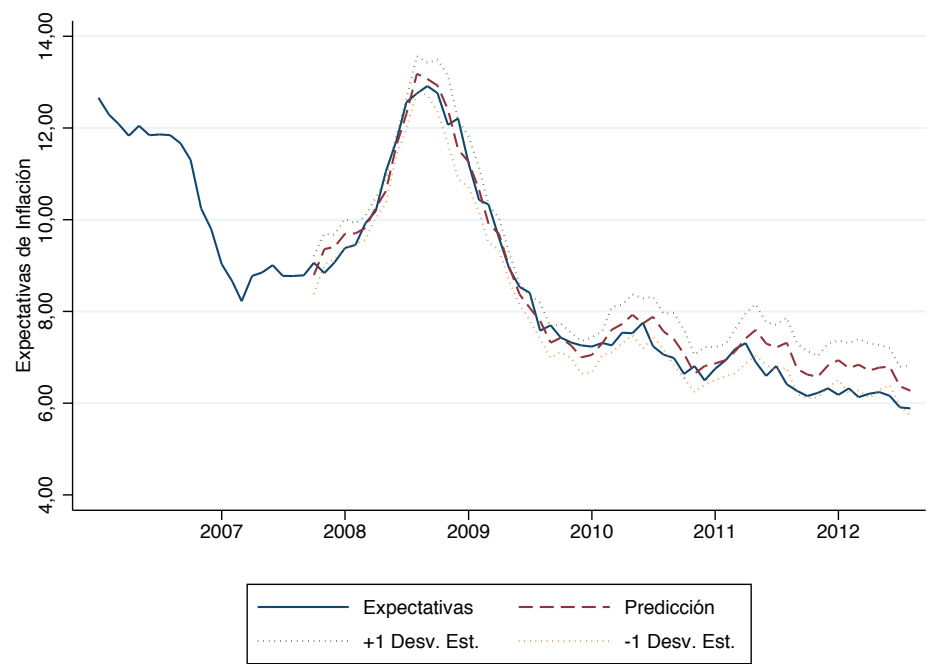

Fuente: Elaboración propia. 